\title{
Diffuse neutral hydrogen in the H I Parkes All Sky Survey ${ }^{\star}$
}

\author{
A. Popping ${ }^{1,2}$ and R. Braun ${ }^{3}$ \\ 1 Kapteyn Astronomical Institute, PO Box 800, 9700 AV Groningen, The Netherlands \\ e-mail: attila.popping@icrar.org \\ 2 International Centre for Radio Astronomy Research, The University of Western Australia, 35 Stirling Hwy, Crawley, WA 6009, \\ Australia \\ 3 CSIRO Astronomy and Space Science, PO Box 76, Epping, NSW 1710, Australia
}

Received 27 May 2010 / Accepted 25 July 2011

ABSTRACT

\begin{abstract}
Context. Observations of neutral hydrogen can provide a wealth of information about the distribution and kinematics of galaxies. To learn more about large scale structures and accretion processes, the extended environment of galaxies must also be observed. Numerical simulations predict a cosmic web of extended structures and gaseous filaments.

Aims. To detect $\mathrm{H}_{\mathrm{I}}$ beyond the ionisation edge of galaxy disks, column density sensitivities have to be achieved that probe the regime of Lyman limit systems. Typically H i observations are limited to a brightness sensitivity of $N_{\mathrm{HI}} \sim 10^{19} \mathrm{~cm}^{-2}$ but this has to be improved by at least an order of magnitude.

Methods. In this paper, reprocessed data is presented that was originally observed for the Hi Parkes All Sky Survey (HIPASS). HIPASS provides complete coverage of the region that has been observed for the Westerbork Virgo Filament Hi Survey (WVFS), presented in accompanying papers, and thus is an excellent product for data comparison. The region of interest extends from 8 to 17 hours in right ascension and from -1 to 10 degrees in declination. Although the original HIPASS product already has good flux sensitivity, the sensitivity and noise characteristics can be significantly improved with a different processing method.

Results. The newly processed data has an $1 \sigma \mathrm{rms}$ flux sensitivity of $\sim 10 \mathrm{mJy}$ beam ${ }^{-1}$ over $26 \mathrm{~km} \mathrm{~s}^{-1}$, corresponding to a column density sensitivity of $\sim 3 \times 10^{17} \mathrm{~cm}^{-2}$. While the rms sensitivity is improved by only a modest $20 \%$, the more substantial benefit is in the reduction of spectral artefacts near bright sources by more than an order of magnitude. In the reprocessed region we confirm all previously catalogued HIPASS sources and have identified 29 additional sources of which 14 are completely new H i detections. We derived spectra and moment maps for all detections together with total fluxes determined both by integrating the spectrum and by integrating the flux in the moment maps within the source radius. Extended emission or companions were sought in the nearby environment of each discrete detection. Ten extra-galactic filaments are marginally detected within the moment maps.

Conclusions. With the improved sensitivity after reprocessing and its large sky coverage, the HIPASS data is a valuable resource for detection of faint $\mathrm{H}_{\mathrm{I}}$ emission. This faint emission can correspond to extended halos, dwarf galaxies, tidal remnant and potentially diffuse filaments that represent the trace neutral fraction of the Cosmic Web.
\end{abstract}

Key words. galaxies: formation - galaxies: halos - intergalactic medium

\section{Introduction}

Current cosmological models ascribe about $4 \%$ of the density to baryons (Spergel et al. 2007). At low redshift most of these baryons do not reside in galaxies, but are expected to be hidden in extended gaseous web-like filaments (e.g. Davé et al. 1999; Davé et al. 2001; Cen \& Ostriker 1999). Calculations sugget that in the current epoch baryons are almost equally distributed amongst three components: (1) galactic concentrations; (2) a warm-hot intergalactic medium (WHIM) and (3) a diffuse intergalactic medium (seen as the $\operatorname{Ly} \alpha$ forest). These simulations predict that the three components are each coupled to a decreasing range of baryonic overdensity $\log \left(\rho / \bar{\rho}_{\mathrm{H}}\right)>3.5,1-3.5$, and $<3.5$.

Direct detection of the inter-galactic gas, or the WHIM, is very difficult in the EUV and X-ray bands (Cen \& Ostriker 1999). In this and accompanying papers we make an effort to detect traces of the inter and circum-galactic medium in neutral hydrogen. Due to the moderately high temperatures in the intergalactic medium (above $10^{4}$ Kelvin), most of the gas in the

* Appendix $\mathrm{A}$ is available in electronic form at http://www . aanda.org
Cosmic Web is highly ionised. To detect the trace neutral fraction in the Lyman Limit Systems using the 21-cm line of neutral hydrogen, a column density sensitivity of $N_{\mathrm{HI}} \sim 10^{17-18}$ is required. A more detailed background and introduction to this topic is given in Popping (2010) and Popping \& Braun (2011a).

A first example of detection in $\mathrm{H}_{\mathrm{I}}$ emission of the likely counterpart of a Lyman Limit absorption System is shown in Braun \& Thilker (2004), where a very diffuse H I structure is seen with a peak column density of only $N_{\mathrm{HI}} \sim 10^{18} \mathrm{~cm}^{-2}$, connecting M 31 and M 33. To be able to detect a large number of diffuse $\mathrm{H}_{\mathrm{I}}$ features, extended blind surveys are required with an excellent brightness sensitivity. One of the first such efforts is presented in Popping \& Braun (2011a), where the Westerbork Synthesis Radio Telescope (WSRT) is used to undertake a deep fully-sampled survey of the galaxy filament joining the Local Group to the Virgo Cluster (Westerbork Virgo Filament H I Survey) extending from 8 to $17 \mathrm{~h}$ in RA and from -1 to +10 degrees in Declination. Data products were created from both the cross-, as well as the auto-correlation data, to achieve a very high brightness sensitivity at a variety of spatial resolutions. The total-power product of the WVFS is presented in Popping \& Braun (2011a), while the interferometric 
data is presented in Popping \& Braun (2011b). In these papers new detections of neutral hydrogen are reported. Although these detections are very interesting, they are difficult to interpret or to confirm, as no comparison data is currently available at a comparable sensitivity.

In this paper we use reprocessed data of the Hi Parkes All Sky Survey (HIPASS) to complement the WVFS observation. The HIPASS (Barnes et al. 2001) includes the complete Southern sky and the Northern sky up to +25.5 degrees in Declination. The Northern part of the survey is described in Wong et al. (2006). This survey currently has the best available H I brightness sensitivity yet published. As the Northern part of the survey completely covers the region that has been observed for the Westerbork Virgo Filament Survey (WVFS), HIPASS is an excellent product for data comparison.

Although neither the flux sensitivity, nor the brightness sensitivity of HIPASS is equivalent to that of the WVFS total power data, we can still learn more about faint $\mathrm{H}_{\mathrm{I}}$ detections in the WVFS, by taking into account the limitations of both surveys. The low column densities of some new $\mathrm{H}_{\mathrm{I}}$ detections in the WVFS might be confirmed, indicating that the gas is indeed very diffuse. Conversely, if column densities measured in the HIPASS data are significantly higher than in the WVFS, this would imply that the gas is more condensed than it appeared, with the emission diluted by the large beam of the WVFS.

Although the HIPASS data is completely reduced and the processed cubes are publicly available, for our purpose we have begun anew with the raw, unprocessed observational data. Increased computing capacity, and different calibration algorithms allow significant improvements to be achieved over the original HIPASS products.

In the observations and data reduction sections we will explain in detail the processing employed and the improvements achieved. A new list of objects detected in the region of interest is given in the results section. Although the improved data reduction method can be applied to the complete HIPASS survey area, we emphasise that we have only applied it to the region of overlap with the WVFS both spatially and spectrally.

In Sect. 2 of this paper we will briefly summarise the observations that have been used, followed by the data reduction strategy in Sect. 3. In Sect. 4 the results will be presented; the general properties of each detected object are given, but new $\mathrm{H}_{\text {I }}$ detections in the HIPASS data are discussed in more detail. We close with a short discussion and conclusion in Sects. 5 and 6. Detailed analysis and comparison of the data, together with a discussion of the nature of the $\mathrm{HI}_{\mathrm{I}}$ emission will be presented in a future paper. In that paper we will compare the results of the HIPASS data together with the auto- and cross-correlation products of the WVFS.

\section{Observations}

In our search for diffuse Hi emission, we have employed data that was originally acquired for the HIPASS. The data is described in detail in Barnes et al. (2001) and we will only summarise the relevant properties. All data has been obtained using the Parkes 21-cm Multibeam system, containing a cooled 13 beam receiver and digital correlator. The Multibeam correlator has an instantaneous bandwidth of $64 \mathrm{MHz}$ divided into 1024 channels. For the HIPASS observations the receivers were tuned to a central frequency of $1394.5 \mathrm{MHz}$, offering a velocity range of -1280 to $12700 \mathrm{~km} \mathrm{~s}^{-1}$ with a mean channel separation of $13.4 \mathrm{~km} \mathrm{~s}^{-1}$. The central beam FWHM is 14.0 arcmin, and the 13 beams are separated from one another by about
30 arcmin. Data acquisition was started in 1997 February and completed in 2000 March. Observations were obtained by scanning the Telescope in Declination strips of 8 degrees length. The multibeam receiver is rotated relative to the scan direction, to get approximately uniformly spaced sampling of the sky over a strip of $\sim 1.7$ degrees width. Each Declination scan maps approximately $8 \times 1.7$ degrees. To obtain full coverage of the sky at full sensitivity, subsequent scans are displaced by 7 arcmin in RA, which means that each of the 13 beams maps the sky with Nyquist sampling. The scan rate of each strip is 1 degree $\mathrm{min}^{-1}$. Using all 13 beams, the total integration time of the HIPASS survey results in $7 \times 10^{3} \mathrm{~s} \mathrm{deg}^{-2}$, or $450 \mathrm{~s} \mathrm{beam}^{-1}$. The typical sensitivity of the original HIPASS product is $13.3 \mathrm{mJy}^{\text {beam }^{-1}}$ over $18 \mathrm{~km} \mathrm{~s}^{-1}$.

For the original HIPASS product, cubes were created of $8 \times 8$ degrees in size, centered at Declinations between -90 and +24 degrees. For our purpose we have selected all original HIPASS scans centered at a Declination of $-2,6$ and 14 degrees, and between 8 and $17 \mathrm{~h}$ in Right Ascension. With these data we achieve the best possible coverage and sensitivity in the region between -1 and 10 degrees in Declination. This region was selected to exactly overlap with the region observed in the WVFS. The WVFS is an unbiased survey of $\sim 1500$ squared degrees, undertaken with the WSRT, directed at the galaxy filament connecting the Local Group with the Virgo Cluster. The WVFS total power data has an effective beam size of $\sim 49$ arcmin with a sensitivity of $16 \mathrm{mJy}$ beam ${ }^{-1}$ over $16 \mathrm{~km} \mathrm{~s}^{-1}$. The HIPASS data has a slightly superior flux sensitivity, but because of the smaller beam size the column density sensitivity is about an order of magnitude worse. Nevertheless the HIPASS data is an excellent product to use for comparison with the WVFS data.

\section{Data reduction}

\subsection{Bandpass removal in the original HIPASS product}

Although the original unprocessed HIPASS data have been used, the reduction method is slightly different, to obtain an improved end product. The most challenging aspect of calibrating an observed total power spectrum is the accurate estimation of the system bandpass shape. Bandpass calibration of a single dish telescope is traditionally accomplished by observing in signal/reference mode. The telescope alternately tracks the target position and a suitable nearby reference region for the same amount of time. The reference position is used to estimate the bandpass shape and is divided out of the signal spectrum. For HIPASS the telescope was scanning the sky continuously, so the straight-forward signal/reference method could not be employed. The method that has been used, was to estimate the bandpass shape of each spectrum, by using a combination of earlier and later spectra, observed by the same feed of the multibeam receiver. The bandpass was estimated by taking a channel-by-channel median of the earlier and later spectra. The median reference spectrum was preferred above the mean reference spectrum, as the median statistic is more robust to outlying data points, and is independent of the magnitude of deviation of outlying points.

The strategy that has been used in reducing the raw HIPASS data works well in the absence of line emission, but breaks down in the vicinity of bright detections. Some $\mathrm{H}$ I sources are sufficiently bright that bandpass estimates just prior and after the target spectrum are elevated by the source itself. This results in negative artefacts, or bandpass sidelobes that 
appear as depressions in the spectra north and south of strong Hi sources.

\subsection{Bandpass removal in reprocessed HIPASS data}

All unprocessed HIPASS spectra are archived and can be reprocessed using different methods. Techniques can be developed to improve the bandpass-sidelobes and preserve spatially extended emission. An example of such an approach is given by Putman et al. (2003) where a different processing algorithm has been employed to image HVCs and the Magellanic Stream. We have tested many different algorithms, including the original HIPASS processing pipeline and the method used by Putman et al. (2003), and achieved the lowest residual rms fluctuation level with the approach outlined below.

The data were bandpass-corrected, calibrated and Dopplertracked using the aips ++ program LiveData (Barnes et al. 2001) in the following manner.

- The spectra were hanning smoothed over three channels to a velocity resolution of $\sim 26 \mathrm{~km} \mathrm{~s}^{-1}$.

- In estimating the shape of the bandpass, a complete $8^{\circ}$ scan is used instead of just a few time steps before and after the target spectrum. By using a complete scan instead of a subset the statistics are improved, making the bandpass estimate more robust.

- A third order polynomial has been fit to the data in the time domain. Data points outside 2 times the standard deviation were excluded from the polynomial fit. This process is iterated three times, to get the best possible outlier rejection.

- After fitting and correcting the data in the time domain, a second order polynomial was fit in the frequency domain. Higher order polynomials in frequency were tested but did not improve the result.

All the processed scans were gridded with Gridzilla (Barnes et al. 2001) using a pixel size of $4^{\prime}$. Cubes were created with a size of 24 degrees in Declination, ranging from -6 to 18 degrees and typical width of $1 \mathrm{~h}$ in Right Ascension with an overlap of one degree between the adjacent cubes.

All overlapping scans were averaged using the system temperature weighted median of relevant data points. The value of the median is strongly dependent on the form of the weighting function $w(r)$ and the radius $r_{\max }$ out to which spectra are included. The weighting procedure for HIPASS is described in detail in Barnes et al. (2001). A Gaussian beam-shape is assumed, so the weighting function has the functional form:

$w(r)= \begin{cases}\exp \left[-\left(\frac{r}{\sigma}\right)^{2} / 2\right] & \text { for } r \leq r_{\max } \\ 0 & \text { for } r>r_{\max } .\end{cases}$

For gridding the HIPASS data, a value of $r_{\max }=6$ arcmin has been adopted. The estimated flux at a given pixel is determined by the weighted median of all spectra contributing to that pixel and has the form:

$F_{e}=\frac{\operatorname{median}(F)}{\operatorname{median}(w)}$

For a random distribution of data points or observations, the median of the weights $[$ median $(w)]$ is determined by the weighting function where the radius divides the smoothing area in two equivalent parts, i.e. $w\left(r_{\max } / \sqrt{2}\right)$. For the adopted $r_{\max }$ of $6 \operatorname{arcmin}, \operatorname{median}(w)=1.28$, which has been taken into account when gridding the data. Tests during the gridding of HIPASS data have shown that the input spectra are very nearly randomly distributed on the sky (Barnes et al. 2001).

A top-hat kernel of $12^{\prime}$ has been used to smooth data spatially. The final beam size of the gridded data cubes is approximately $15.5^{\prime} F W H M$, although as discussed at some length in Barnes et al. (2001), the effective beam-size is dependent on the signal-to-noise ratio of a detection.

After the processed cubes were formed, sub-cubes were created using the inner 14 degrees in Declination with the highest uniform sensitivity overlapping with the WVFS data. A velocity range was selected from 200 to $1700 \mathrm{~km} \mathrm{~s}^{-1}$, again to match with the velocity coverage of the WVFS data.

In the reprocessed data, we typically achieve an rms flux sensitivity of $\sim 10 \mathrm{mJy}$ beam ${ }^{-1}$ over $26 \mathrm{~km} \mathrm{~s}^{-1}$. This is an improvement on the original HIPASS processing, although we have degraded the velocity resolution to $26 \mathrm{~km} \mathrm{~s}^{-1}$ compared to $18 \mathrm{~km} \mathrm{~s}^{-1}$, due to the Hanning smoothing that has been used instead of the Tukey smoothing. When scaling both noise values to the same velocity resolution, the achieved sensitivity is a significant improvement on the sensitivity of the first HIPASS product. The typical sensitivity of the first HIPASS product is $\sim 13 \mathrm{mJy}$ beam $^{-1}$ over $18 \mathrm{~km} \mathrm{~s}^{-1}$. For the northern Declinations we are concentrating on, the sensitivity is slightly worse at $\sim 14 \mathrm{mJy}_{\text {beam }}^{-1}$ over $18 \mathrm{~km} \mathrm{~s}^{-1}$ (Wong et al. 2006) corresponding to $\sim 12 \mathrm{mJy}$ beam ${ }^{-1}$ over $26 \mathrm{~km} \mathrm{~s}^{-1}$. The previous reprocessing of the HIPASS data (Putman et al. 2003) resulted in a similar rms value as we are achieving, however was not able to correct for the negative artefacts. Rather than an improved rms value, the more important benefit of reprocessing the data is that the negative artefacts in the vicinity of bright sources are suppressed by more than an order of magnitude.

An example of the reduced data and its artefacts is given in Fig. 1. The left panel shows a region of the sky processed with the original HIPASS pipeline. There are strong negative artefacts north and south of strong H I sources. The right panel of Fig. 1 shows the same region processed with the improved reduction pipeline. Although the spectral artefacts are still visible, there is a dramatic improvement. The reprocessed data will be much more sensitive to diffuse $\mathrm{H}_{\mathrm{r}}$ emission, especially in the direct vicinity of bright $\mathrm{H}_{\mathrm{I}}$ objects. Using this follow-up HIPASS product, better flux estimates can be determined for discrete sources. Moreover, it also allows investigation of the nearby environment of discrete bright objects, which has previously been impossible.

\section{Results}

A significant sky area of more than $1500 \mathrm{deg}^{2}$ has been reprocessed from the original HIPASS data. We achieve an rms sensitivity that is improved by $\sim 20 \%$ compared to the published HIPASS product. More important than this improvement in noise, is the very effective suppression of the negative artefacts surrounding all bright $\mathrm{H}$ i detections. We expect slightly different flux values, especially for the bright $\mathrm{H}_{\text {I }}$ objects, as the newly processed data is more sensitive to extended emission. Furthermore, we expect to have more $\mathrm{H}_{\mathrm{I}}$ detections of diffuse or companion galaxies due to the improved sensitivity and artefact suppression.

For the detection of sources we have used two methods. Firstly an automated source finder was employed to identify sources. Developing a fully automated and reliably source finder is extremely difficult, especially when looking for faint objects, therefore all the cubes were also inspected visually to identify features that might be missed by the automated source finder. 

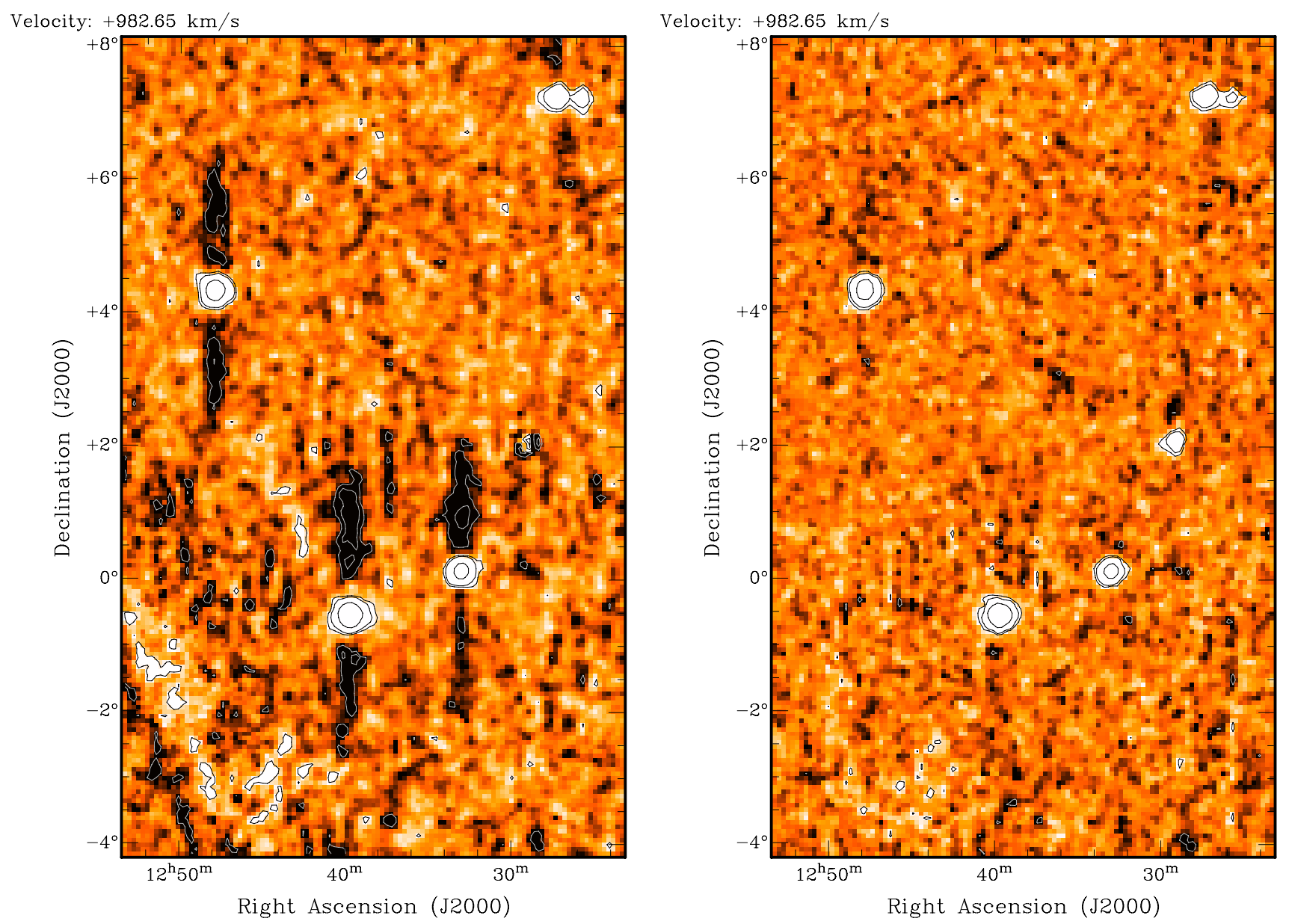

Fig. 1. Example of the artefacts in the original HIPASS pipeline (left panel) and the reprocessed data (right panel). Both panels show the same region of the sky at the same velocity, the intensity ranges from -40 to 40 mJy beam ${ }^{-1}$. Contours are plotted at $-60,-30,30,60{\text { and } 300 \mathrm{mJy} \text { beam }}^{-1}$, negative contours are coloured white.

\subsection{Automatic source detection}

We use the Duchamp (Whiting 2008) three dimensional source finding algorithm to identify candidate detections. Features are sought with a peak flux exceeding 5 times the noise value. Detections are grown down from the peak to a cutoff value of three times the noise, to be more sensitive to extended or diffuse emission. The requirement for candidate sources to be accepted is to have a size of at least 10 pixels (after growing) in one channel and to contain pixels from at least 2 adjacent channels. As a result the minimum velocity width of a detection is $26 \mathrm{~km} \mathrm{~s}^{-1}$ which is sufficient to detect most galaxies, but low mass dwarf galaxies or companions might be missed. The search criteria were chosen to eliminate isolated noise peaks and make the detections more reliable.

The original reduced data has a velocity resolution of $26 \mathrm{~km} \mathrm{~s}^{-1}$. Hanning smoothed versions of the data cubes have been created at a velocity resolution of 52 and $104 \mathrm{~km} \mathrm{~s}^{-1}$. These smoothed cubes are more sensitive to structures that are extended in the velocity domain. Duchamp was applied in a similar way as to the original data cubes, however this did not result in additional detections.

Spectra and moment maps of all the candidate detections have been inspected visually for reliability. Strong artefacts due to e.g. solar interference can be easily identified in the integrated maps and these features were eliminated from the source list. The spectra can be used to identify false detections that are caused by ripples in the bandpass seen toward continuum sources.

\subsection{Visual source detection}

Although automatic source finders are an excellent tool to search for candidate detections they have their limitations. Detecting point sources is relatively easy, as they are clearly defined in both the spatial and velocity domain. We are especially interested in diffuse features that have a peak brightness of only a few times the noise. These sources can only be found when source finders are "tuned" to the actual properties, the spatial and spectral extent, of these features. Since we do not know the appearance or existence of the sources beforehand, it is difficult to employ dedicated source finders. Another complication is that the noise is not completely uniform. The noise can be elevated, for example, in the vicinity of continuum sources or in the case of solar interference. Several efforts have been made to look for diffuse but very extended features, both spatially and in velocity. Unfortunately these efforts have not been very successful and resulted in previously known or unreliable detections.

Visual inspection of the data was necessary, to have a better understanding of the quality and features in the data, but also to look for features that have been missed by the automated source finder. All cubes were inspected in the spatial domain as well as in the two velocity domains (RA versus velocity and Dec versus velocity) to search for objects that had been missed. 


\subsection{Detected sources}

For each candidate detection an optical counterpart was sought in the NASA Extragalactic Database (NED) ${ }^{1}$ within a search radius of 14 arcmin. This radius is approximately the diameter of the HIPASS beam and only objects within this radius can contribute to the measured flux. Another requirement for potential optical counterparts is to have a known radial velocity that is comparable to the radial velocity of the $\mathrm{H}$ i detection. Candidate detections with a clearly identified optical counterpart are accepted. For each candidate detection the noise is determined based on the line-width at $20 \%$ of the peak $\left(W_{20}\right)$. For line-widths smaller than $250 \mathrm{~km} \mathrm{~s}^{-1}$ the noise is given as:

$n=\sqrt{\frac{1.5 W_{20}}{v_{\mathrm{res}}}} \cdot \mathrm{rms} \cdot v_{\mathrm{res}}$

where $n$ is in units of $\left[\mathrm{Jy} \mathrm{km} \mathrm{s}^{-1}\right]$, rms is the sensitivity of the cubes and $v_{\text {res }}$ is the velocity resolution. For broader profiles the term $\left(1.5 W_{20}\right)$ is replaced by $\left(W_{20}+50 \mathrm{~km} \mathrm{~s}^{-1}\right)$ to account for the line wings.

Using this noise measurement, a signal-to-noise ratio can be determined for each candidate detection. Candidate detections with an integrated signal-to-noise larger than 5 are accepted as detections.

Finally, a list of 203 detections was obtained of which 31 were detected by visual inspection; all detections and their observed properties are listed in Table 1 . The first column gives the catalogue name, composed of the three characters "HIR", an abbreviation of "HIPASS Reprocessed", followed by two sets of four digits indicating the Right Ascension and Declination of the detection. The second column gives the optical identification of the detection if any is known and the third column gives the original HIPASS ID if available. Completely new $\mathrm{H}_{\mathrm{I}}$ detections are indicated in the third column by "new". In the case a detections is not mentioned in the HIPASS catalogue however is detected by ALFALFA, this is indicated with " $\alpha \alpha$ ". The fourth, fifth and sixth column give the spatial position and heliocentric velocity of the source. This is followed by velocity width of the object, at $20 \%$ of the peak flux. The seventh and eighth columns give the integrated flux (3D) and the integrated line-strength (1D) respectively. We will discuss the differences between the two different flux measurements in the following section. The last column indicates whether the object is detected by the automatic source finder (A) or by visual inspection (V). In some cases the objects are completely at the edge of the processed bandwidth. Although a detection here can be a solid detection, the estimated flux and line-width values are underestimated as a part of the spectrum is missing. For objects at the edge of the bandwidth, this is indicated in the table with a letter " $(u)$ ".

\subsection{New HI detections}

All sources in the HIPASS catalogue have been re-detected in the reprocessed HIPASS product. Apart from these previously known Hi detections, 29 detections have been made that were not listed in the HIPASS catalogue, of which 14 are completely new $\mathrm{H}_{\mathrm{I}}$ detections. Thirteen of the detections that were not listed in the original HIPASS catalogue were recovered through visual inspection. Amongst the detections that are not listed in

\footnotetext{
1 The NASA/IPAC Extragalactic Database (NED) is operated by the Jet Propulsion Laboratory, California Institute of Technology, under contract with the National Aeronautics and Space Administration.
}

the HIPASS catalogue, 6 objects have since been detected in the ALFALFA survey and are listed in the third data catalog (Kent et al. 2008) covering right ascensions from 11:36 to $13: 52 \mathrm{~h}$ and declinations form +8 to +12 degrees. In fact, all the newly detected HIPASS objects within this area can be confirmed with the ALFALFA catalogue.

Spectra of completely new H I detections are shown in Fig. 2. The spectra of the new detections do not show some particular feature and they are both found by visual ( 8 detections) and automated ( 6 detections) inspection. Only the velocity interval that overlaps with the velocity coverage of the WVFS survey, from $\sim 400$ to $\sim 1800 \mathrm{~km} \mathrm{~s}^{-1}$, has been reprocessed. As the bandwidth of the reprocessed data is relatively small compared to the full HIPASS frequency coverage, detections at the edge of the reprocessed data appear at the very edge in Fig. 2.

HIR 0859+1109: this is a new H I detection for which no optical galaxy is known at the relevant radial velocity. At a radial velocity of $1988 \pm 40 \mathrm{~km} \mathrm{~s}^{-1}$ and with an offset of 1.6 arcmin is UGC 4712, which is more than $300 \mathrm{~km} \mathrm{~s}^{-1}$ higher than the radial velocity of HIR $0859+1109$. It is possible that HIR $0859+1109$ is the Hi counterpart of UGC 4712.

HIR 0911+0024: when looking at the spectrum of this detection, there is one narrow peak that looks significant. There is no optical galaxy known at this redshift.

HIR 0921+0725: the nature of this H I detection is not clear. The DSS image shows a diffuse feature at a location of RA = 09:21:26.3 and Dec $=07: 21: 57$, but many SDSS objects are listed at this location, all without any distance information. Based on the appearance of the optical feature, we expect that all these are at a higher redshift and not related to HIR 0921+0725.

HIR 0946+0141: this H I feature is very likely the counterpart of SDSS J094602.54+014019.4, a spiral galaxy at a radial velocity of $1753 \mathrm{~km} \mathrm{~s}^{-1}$. Both the radial velocity and the DSS image are well-matched to the H I detection.

HIR 1005+0139: at a spatial separation of only 1.5 arcmin is 2dFGRS N421Z115 with a radial velocity within $30 \mathrm{~km} \mathrm{~s}^{-1}$ of HIR 1005+0139. This detection is a completely new H I detection and is the H I counterpart of 2dFGRS N421Z115 with high certainty.

HIR 1052+0002: when inspecting the DSS image, a small galaxy can be identified at the peak of the $\mathrm{H}_{\mathrm{I}}$ contours. This is the irregular galaxy MGC 0013223 at a radial velocity of $1772 \mathrm{~km} \mathrm{~s}^{-1}$, which is very similar to the observed H I radial velocity. Although $\mathrm{HI}_{\mathrm{I}}$ has not been observed before in this galaxy, HIR $1052+0002$ is very likely the neutral counterpart of MGC 0013223.

HIR 1055+0511: although the peak of this detection is not very bright, the line is broad enough to make it significant. The DSS image shows a diffuse object at RA $=10: 55: 16.3$ and Dec $=05: 12: 19.5$ that might be relevant for this candidate detection. However, this is an SDSS object at a cataloged radial velocity of almost $6000 \mathrm{~km} \mathrm{~s}^{-1}$. If correct, any relation with HIR $1055+0511$ is highly unlikely.

HIR 1212+0248: less than half an arcmin separated and at exactly the same radial velocity is LEDA 135791. HIR $1212+0248$ is the first $\mathrm{H}$ I detection of this dwarf irregular galaxy.

HIR 1230+0013: this feature is about 30 arcmin separated from NGC 4517A. Although there is no sign of any optical counterpart in the DSS images, HIR $1230+0013$ is possibly associated with NGC 4517A, as the radial velocity is very similar.

HIR 1231+0145: at an angular offset of less than 5 arcmin and a similar radial velocity is the irregular galaxy CGCG 014054. Although this galaxy has no reported H I, HIR 1231+0145 
Table 1. Observed properties of H I detections in reprocessed HIPASS data within the Westerbork Virgo Filament Survey region.

\begin{tabular}{|c|c|c|c|c|c|c|c|c|c|}
\hline Name & Optical ID. & HIPASS ID & RA [hh:mm:ss] & Dec [dd:mm:ss] & $V_{\mathrm{Hel}}^{a}$ & $W_{20}^{b}$ & $S_{\text {int }^{c}}$ & $S_{\text {line }}^{d}$ & det. \\
\hline HIR 0821-0025 & UGC 04358 & HIPASS J0821-00 & $8: 21: 41$ & $-0: 25: 00$ & $1775^{u}$ & $50^{u}$ & $4.1^{u}$ & $3.5^{u}$ & A \\
\hline HIR 0859+1109 & UGC 04712 & new & $8: 59: 28$ & 11:09:23 & 1643 & 80 & 1.9 & 3.0 & A \\
\hline HIR 0906+0618 & UGC 04781 & HIPASS J0906+06 & 9:06:36 & $6: 18: 27$ & 1433 & 168 & 19.7 & 16.0 & A \\
\hline HIR 0908+0555 & UGC 04797 & HIPASS J0908+05a & $9: 08: 12$ & $5: 55: 54$ & 1326 & 102 & 5.6 & 5.3 & A \\
\hline HIR 0908+0517 & SDSS J090836.54+051726.8 & HIPASS J0908+05b & $9: 08: 43$ & $5: 17: 49$ & 600 & 57 & 1.3 & 2.2 & A \\
\hline HIR 0910+0711 & NGC 2777 & HIPASSJ0910+07 & $9: 10: 34$ & $7: 11: 43$ & 1500 & 139 & 11.6 & 9.9 & A \\
\hline HIR 0911+0024 & No object found. & new & $9: 11: 21$ & $0: 24: 59$ & 1286 & 83 & 2.8 & 2.7 & A \\
\hline HIR 0921+0725 & No object found. & new & 9:21:07 & $7: 25: 32$ & 1369 & 110 & 3.7 & 4.5 & A \\
\hline HIR 0944-0038 & UGC 05205 & HIPASS J0944-00a & 9:44:06 & $-0: 38: 42$ & 1485 & 178 & 15.0 & 13.1 & A \\
\hline HIR 0944+0937 & IC 0559 & HIPASS J0944+09 & 9:44:31 & 9:37:06 & 522 & 132 & 5.6 & 4.8 & A \\
\hline HIR 0944-0040 & SDSS J094446.23-004118.2 & HIPASS J0944-00b & $9: 44: 43$ & $-0: 40: 37$ & 1222 & 161 & 7.0 & 8.5 & A \\
\hline HIR 0946+0141 & SDSS J094602.54+014019.4 & new & 9:46:00 & 1:41:07 & $1763^{u}$ & $53^{u}$ & $1.0^{u}$ & $2.3^{u}$ & A \\
\hline HIR 0946+0031 & UGC 05238 & HIPASS J0946+00 & $9: 46: 55$ & 0:31:09 & $1697^{u}$ & $120^{u}$ & $7.0^{u}$ & $7.7^{u}$ & A \\
\hline HIR 0947+0241 & UGC 05249 & HIPASS J0947+02 & $9: 47: 44$ & $2: 41: 15$ & 1776 & 25 & 1.3 & 1.6 & A \\
\hline HIR 0951+0750 & UGC 05288 & HIPASS J0951+07 & $9: 51: 16$ & $7: 50: 12$ & 555 & 112 & 33.0 & 27.8 & A \\
\hline HIR $0953+0135$ & NGC 3044 & HIPASS J0953+01 & $9: 53: 42$ & $1: 35: 06$ & 1292 & 341 & 47.3 & 49.4 & A \\
\hline HIR 0954+0915 & NGC 3049 & HIPASS J0954+09 & $9: 54: 41$ & $9: 15: 53$ & 1471 & 223 & 6.1 & 9.1 & A \\
\hline HIR 1005+0139 & 2dFGRS N421Z115 & new & $10: 05: 20$ & 1:39:48 & 1260 & 95 & 2.0 & 2.4 & V \\
\hline HIR $1007+1022$ & UGC 05456 & HIPASS J1007+10 & $10: 07: 18$ & $10: 22: 18$ & 547 & 90 & 4.8 & 7.2 & A \\
\hline HIR 1013+0702 & UGC 05522 & HIPASS J1013+07 & $10: 13: 57$ & $7: 02: 36$ & 1219 & 232 & 44.7 & 42.8 & A \\
\hline HIR 1014+0329 & NGC 3169 & HIPASS J1014+03 & $10: 14: 17$ & $3: 29: 28$ & 1246 & 457 & 141.4 & 98.7 & $\mathrm{~A}$ \\
\hline HIR $1015+0242$ & UGC 05539 & HIPASS J1015+02 & $10: 15: 52$ & $2: 42: 11$ & 1275 & 157 & 11.4 & 13.7 & A \\
\hline HIR 1017+0421 & UGC 05551 & HIPASS J1017+04 & 10:17:12 & $4: 21: 37$ & 1340 & 90 & 9.4 & 6.0 & A \\
\hline HIR $1028+0335$ & UGC 05677 & HIPASS J1028+03 & $10: 28: 33$ & $3: 35: 28$ & 1146 & 139 & 3.2 & 5.4 & A \\
\hline HIR $1031+0428$ & UGC 05708 & HIPASS J1031+04 & $10: 31: 15$ & $4: 28: 27$ & 1169 & 198 & 33.9 & 33.9 & A \\
\hline HIR $1038+1024$ & CGCG 065-074 & HIPASS J1038+10 & $10: 38: 14$ & $10: 24: 26$ & 1167 & 192 & 8.1 & 6.9 & A \\
\hline HIR 1039+0145 & UGC 05797 & HIPASS J1039+01 & $10: 39: 28$ & $1: 45: 15$ & 706 & 71 & 3.9 & 3.0 & A \\
\hline HIR 1044+1134 & MESSIER 095 & HIPASS J1044+11 & 10:44:02 & $11: 34: 18$ & 798 & 280 & 12.2 & 32.8 & A \\
\hline HIR 1046+0149 & NGC 3365 & HIPASS J1046+01 & 10:46:11 & 1:49:32 & 985 & 248 & 36.1 & 43.1 & A \\
\hline HIR $1051+0550$ & NGC 3423 & HIPASS J1051+05 & $10: 51: 17$ & $5: 50: 24$ & 1010 & 186 & 42.4 & 45.8 & A \\
\hline HIR $1051+0327$ & HIPASS J1051+03 & HIPASS J1051+03 & $10: 51: 31$ & $3: 27: 20$ & 1062 & 85 & 13.0 & 13.3 & A \\
\hline HIR $1051+0435$ & UGC 05974 & HIPASS J1051+04 & $10: 51: 34$ & $4: 35: 53$ & 1050 & 175 & 11.1 & 15.3 & A \\
\hline HIR $1052+0002$ & MGC 0013223 & new & $10: 52: 49$ & $0: 02: 35$ & $1776^{u}$ & $30^{u}$ & $1.3^{u}$ & $1.2^{u}$ & A \\
\hline HIR $1053+0232$ & LSBC L1-137 & HIPASS J1053+02 & $10: 53: 08$ & $2: 32: 41$ & 1035 & 89 & 6.4 & 6.4 & A \\
\hline HIR $1055+0511$ & No object found. & new & $10: 55: 27$ & $5: 11: 57$ & 982 & 142 & 6.4 & 3.8 & V \\
\hline HIR 1101+0338 & NGC 3495 & HIPASS J1101+03 & $11: 01: 15$ & $3: 38: 01$ & 1119 & 324 & 27.4 & 30.5 & A \\
\hline HIR 1105-0002 & NGC 3521 & HIPASS J1105-00 & $11: 05: 48$ & $-0: 02: 05$ & 793 & 444 & 298.8 & 199.0 & A \\
\hline HIR 1107+0710 & NGC 3526 & HIPASS J1106+07 & 11:07:00 & 7:10:54 & 1422 & 194 & 10.6 & 6.6 & A \\
\hline HIR 1110+0107 & CGCG 011-018 & HIPASS J1110+01 & $11: 10: 56$ & $1: 07: 45$ & 983 & 96 & 3.4 & 5.1 & $\mathrm{~A}$ \\
\hline HIR 1112+1014 & UGC 06248 & HIPASS J1112+10 & $11: 12: 45$ & $10: 14: 03$ & 1286 & 75 & 1.8 & 2.7 & $\mathrm{~A}$ \\
\hline HIR 1117+0434 & NGC 3604 & HIPASS J1117+04 & $11: 17: 30$ & $4: 34: 25$ & 1511 & 209 & 7.9 & 10.8 & A \\
\hline HIR 1119+0939 & SDSS J111928.10+093544.2 & HIPASS J1119+09 & 11:19:46 & $9: 39: 12$ & 1075 & 298 & 4.6 & 3.6 & $\mathrm{~V}$ \\
\hline HIR 1120+0232 & UGC 06345 & HIPASS J1120+02 & 11:20:11 & $2: 32: 48$ & 1604 & 141 & 19.7 & 22.0 & A \\
\hline HIR $1124+0318$ & NGC 3664 & HIPASS J1124+03 & $11: 24: 25$ & $3: 18: 44$ & 1379 & 140 & 20.5 & 22.7 & A \\
\hline HIR 1124+1121 & NGC 3666 & HIPASS J1124+11 & $11: 24: 25$ & $11: 21: 16$ & 1056 & 278 & 41.0 & 45.9 & A \\
\hline HIR $1125+0958$ & IC 0692 & HIPASS J1126+10 & $11: 25: 53$ & 9:58:09 & 1154 & 102 & 3.1 & 3.5 & A \\
\hline HIR $1127+0846$ & IC 2828 & HIPASS J1127+08 & $11: 27: 03$ & 8:46:06 & 1048 & 79 & 1.0 & 1.6 & V \\
\hline HIR 1127-0058 & UGC 06457 & HIPASS J1127-00 & $11: 27: 10$ & $-0: 58: 30$ & 956 & 106 & 7.2 & 7.9 & A \\
\hline HIR $1128+0923$ & NGC 3692 & HIPASS J1128+09a & $11: 28: 19$ & $9: 23: 35$ & 1617 & 236 & 11.7 & 9.4 & A \\
\hline HIR $1130+0917$ & NGC 3705 & HIPASS J1130+09 & $11: 30: 02$ & $9: 17: 38$ & 1018 & 353 & 29.6 & 34.4 & A \\
\hline HIR 1136+0049 & UGC 06578 & HIPASS J1136+00b & $11: 36: 32$ & 0:49:04 & 1099 & 95 & 5.5 & 5.7 & A \\
\hline HIR $1140+1128$ & NGC 3810 & HIPASS J1140+11 & 11:40:59 & 11:28:07 & 995 & 268 & 33.6 & 38.5 & A \\
\hline HIR 1144+0210 & SDSS J114454.28+020946.8 & HIPASS J1145+02 & 11:44:52 & $2: 10: 15$ & 1009 & 52 & 4.1 & 3.5 & A \\
\hline HIR 1158-0127 & UGC 06970 & HIPASS J1158-01 & $11: 58: 41$ & $-1: 27: 28$ & 1471 & 166 & 46.4 & 4.4 & A \\
\hline HIR 1200-0105 & NGC 4030 & HIPASS J1200-01 & $12: 00: 26$ & $-1: 05: 52$ & 1465 & 346 & 64.0 & 50.8 & A \\
\hline HIR 1204-0131 & UGC 07053 & HIPASS J1204-01 & $12: 04: 17$ & $-1: 31: 37$ & 1469 & 123 & 6.5 & 8.2 & A \\
\hline HIR 1207+0249 & HIPASS J1208+02 & HIPASS J1208+02 & 12:07:57 & 2:49:05 & 1318 & 220 & 84.7 & 55.7 & A \\
\hline HIR $1211+0201$ & UGC 07178 & HIPASS J1211+02a & $12: 11: 04$ & $2: 01: 37$ & 1334 & 95 & 6.2 & 8.0 & A \\
\hline HIR 1211+0256 & UGC 07185 & HIPASS J1211+02b & $12: 11: 26$ & $2: 56: 53$ & 1300 & 110 & 5.8 & 7.6 & A \\
\hline HIR $1212+0248$ & LEDA 135791 & new & $12: 12: 27$ & $2: 48: 29$ & 877 & 117 & 3.1 & 3.9 & A \\
\hline HIR $1212+1054$ & NGC 4178 & HIPASS J1212+10 & $12: 12: 51$ & $10: 54: 29$ & $476^{u}$ & $56^{u}$ & $10.0^{u}$ & $8.7^{u}$ & A \\
\hline HIR 1214+0747 & UGC 07239 & HIPASS J1214+07 & $12: 14: 12$ & $7: 47: 05$ & 1220 & 148 & 5.3 & 5.3 & A \\
\hline HIR 1214+0911 & VCC 0117 & HIPASS J1214+09 & $12: 14: 48$ & $9: 11: 33$ & $1776^{u}$ & $64^{u}$ & $1.4^{u}$ & $1.8^{u}$ & A \\
\hline HIR $1215+0935$ & NGC 4207 & HIPASS J1215+09a & $12: 15: 26$ & $9: 35: 37$ & 587 & 247 & 3.7 & 4.2 & V \\
\hline HIR 1217+1001 & UGC 07307 & HIPASS J1216+10 & $12: 17: 05$ & 10:01:41 & 1178 & 73 & 6.0 & 5.6 & A \\
\hline
\end{tabular}


Table 1. continued.

\begin{tabular}{|c|c|c|c|c|c|c|c|c|c|}
\hline Name & Optical ID. & HIPASS ID & RA [hh:mm:ss] & Dec [dd:mm:ss] & $V_{\mathrm{Hel}}{ }^{a}$ & $W_{20}^{b}$ & $S_{\text {int }^{c}}^{c}$ & $S_{\text {line }}^{d}$ & det. \\
\hline HIR $1217+0027$ & UGC 07332 & HIPASS J1217+00 & $12: 17: 57$ & $0: 27: 01$ & 932 & 81 & 15.4 & 18.7 & A \\
\hline HIR 1218+0640 & NGC 4241 & HIPASS J1218+06 & $12: 18: 00$ & $6: 40: 59$ & 720 & 126 & 5.4 & 7.3 & A \\
\hline HIR 1219+0639 & VCC 0381 & HIPASS J1219+06b & $12: 19: 50$ & $6: 39: 46$ & $481^{u}$ & $76^{u}$ & $2.0^{u}$ & $2.6^{u}$ & A \\
\hline HIR 1220+0019 & CGCG 014-010 & HIPASS J1220+00 & $12: 20: 10$ & $0: 19: 35$ & 884 & 83 & 2.3 & 2.5 & A \\
\hline HIR $1220+0126$ & UGC 07394 & HIPASS J1220+01 & $12: 20: 34$ & $1: 26: 39$ & 1617 & 226 & 5.5 & 4.7 & $\mathrm{~V}$ \\
\hline HIR 1221+0429 & MESSIER 061 & HIPASS J1221+04 & $12: 21: 51$ & 4:29:23 & 1562 & 180 & 85.1 & 87.2 & A \\
\hline HIR 1222+0434 & NGC 4301 & & $12: 22: 28$ & $4: 34: 36$ & 1267 & 135 & 14.9 & 17.1 & A \\
\hline HIR $1222+0814$ & VCC 0566 & $\alpha \alpha$ & $12: 22: 40$ & $8: 14: 39$ & 1392 & 106 & 4.1 & 2.9 & $\mathrm{~V}$ \\
\hline HIR $1222+1118$ & NGC 4330 & $\alpha \alpha$ & $12: 22: 56$ & $11: 18: 37$ & 1590 & 273 & 5.6 & 7.6 & A \\
\hline HIR $1223+0922$ & NGC 4316 & HIPASS J1222+09 & $12: 23: 01$ & $9: 22: 46$ & 1233 & 321 & 7.3 & 3.8 & $\mathrm{~V}$ \\
\hline HIR $1223+0517$ & NGC 4324 & HIPASS J1223+05 & $12: 23: 15$ & $5: 17: 18$ & 1540 & 131 & 2.9 & 4.1 & A \\
\hline HIR $1224+0636$ & IC 3268 & & $12: 24: 08$ & $6: 36: 20$ & 720 & 129 & 5.9 & 5.3 & $\mathrm{~A}$ \\
\hline HIR 1224+0359 & VCC 0737 & HIPASS J1224+03a & $12: 24: 37$ & $3: 59: 05$ & $1720^{u}$ & $137^{u}$ & $4.2^{u}$ & $3.4^{u}$ & A \\
\hline HIR 1224+0319 & VCC 0739 & HIPASS J1224+03b & $12: 24: 41$ & $3: 19: 30$ & 921 & 68 & 13.8 & 11.4 & A \\
\hline HIR $1225+0545$ & NGC 4376 & HIPASS J1225+05 & $12: 25: 19$ & $5: 45: 19$ & 1141 & 123 & 3.0 & 3.8 & A \\
\hline HIR $1225+0714$ & IC 3322A & HIPASS J1225+07 & $12: 25: 36$ & $7: 14: 11$ & 996 & 298 & 24.6 & 21.0 & $\mathrm{~V}$ \\
\hline HIR $1225+0210$ & UGC 07512 & HIPASS J1225+02 & $12: 25: 42$ & $2: 10: 01$ & 1498 & 78 & 4.5 & 6.2 & A \\
\hline HIR $1225+0548$ & VCC 0848 & HIPASS J1226+05 & $12: 25: 50$ & $5: 48: 50$ & 1531 & 207 & 5.2 & 6.7 & A \\
\hline HIR $1226+1026$ & NGC 4390 & HIPASS J1225+10 & $12: 26: 05$ & $10: 26: 35$ & 1101 & 152 & 4.3 & 2.4 & $\mathrm{~V}$ \\
\hline HIR $1226+0853$ & NGC 4411 & $\alpha \alpha$ & $12: 26: 40$ & $8: 53: 28$ & 1269 & 108 & 24.2 & 21.2 & A \\
\hline HIR 1226+1131 & IC 3356 & HIPASS J1226+11 & $12: 26: 52$ & 11:31:08 & 1097 & 95 & 11.3 & 17.3 & $\mathrm{~A}$ \\
\hline HIR $1226+0230$ & NGC 4409 & HIPASS J1226+02 & $12: 26: 54$ & $2: 30: 21$ & $1680^{u}$ & $193^{u}$ & $9.7^{u}$ & $10.6^{u}$ & A \\
\hline HIR $1226+0800$ & NGC 4416 & $\alpha \alpha$ & $12: 26: 56$ & $8: 00: 26$ & 1392 & 125 & 2.5 & 2.6 & A \\
\hline HIR $1227+0553$ & NGC 4423 & HIPASS J1227+05 & $12: 27: 10$ & $5: 53: 45$ & 1128 & 193 & 10.8 & 10.8 & A \\
\hline HIR $1227+1052$ & IC 3371 & HIPASS J1227+10 & $12: 27: 12$ & $10: 52: 42$ & 930 & 188 & 7.7 & 9.2 & A \\
\hline HIR 1227+0713 & UGC 07557 & HIPASS J1227+07 & $12: 27: 13$ & $7: 13: 56$ & 930 & 172 & 23.2 & 21.2 & $\mathrm{~V}$ \\
\hline HIR 1227+0615 & NGC 4430 & HIPASS J1227+06 & $12: 27: 20$ & $6: 15: 58$ & 1415 & 143 & 3.6 & 5.0 & A \\
\hline HIR 1227+0132 & HI $1225+01$ & HIPASS J1227+01 & $12: 27: 27$ & $1: 32: 48$ & 1286 & 85 & 35.1 & 25.1 & A \\
\hline HIR $1228+0843$ & UGC 07590 & HIPASS J1228+08 & $12: 28: 21$ & $8: 43: 54$ & 1115 & 179 & 8.0 & 8.6 & A \\
\hline HIR 1228+0334 & NGC 4457 & HIPASS J1228+03 & $12: 28: 44$ & $3: 34: 38$ & 890 & 138 & 3.6 & 3.1 & A \\
\hline HIR 1229+0243 & UGC 07612 & & $12: 29: 11$ & $2: 43: 53$ & 1630 & 62 & 4.1 & 4.0 & A \\
\hline HIR 1229+0644 & IC 3414 & HIPASS J1229+06 & $12: 29: 26$ & $6: 44: 45$ & $550^{u}$ & $185^{u}$ & $7.0^{u}$ & $4.6^{u}$ & A \\
\hline HIR $1230+0013$ & No object found. & new & $12: 30: 29$ & $0: 13: 01$ & 1524 & 138 & 2.1 & 3.4 & V \\
\hline HIR 1230+0929 & HIPASS J1230+09 & HIPASS J1230+09 & 12:30:41 & 9:29:04 & $495^{u}$ & $71^{u}$ & $2.8^{u}$ & $2.4^{u}$ & A \\
\hline HIR $1231+0145$ & CGCG 014-054 & new & $12: 31: 02$ & 1:45:09 & 1101 & 52 & 1.6 & 1.2 & $\mathrm{~V}$ \\
\hline HIR 1231+0357 & NGC 4496A & HIPASS J1231+03 & $12: 31: 36$ & $3: 57: 15$ & $1736^{u}$ & $145^{u}$ & $34.2^{u}$ & $43.7^{u}$ & A \\
\hline HIR $1232+0024$ & NGC 4517A & HIPASS J1232+00a & $12: 32: 31$ & $0: 24: 28$ & 1520 & 182 & 36.9 & 35.7 & A \\
\hline HIR $1232+0007$ & NGC 4517 & HIPASS J1232+00b & $12: 32: 43$ & 0:07:30 & 1133 & 325 & 108.9 & 113.5 & A \\
\hline HIR $1233+0436$ & VCC 1468 & & $12: 33: 01$ & $4: 36: 54$ & 1220 & 62 & 0.8 & 1.4 & $\mathrm{~V}$ \\
\hline HIR 1233-0032 & HIPASS J1233-00 & HIPASS J1233-00 & $12: 33: 11$ & $-0: 32: 50$ & 719 & 97 & 1.8 & 2.6 & A \\
\hline HIR $1233+0840$ & NGC 4519 & HIPASS J1233+08 & $12: 33: 26$ & $8: 40: 24$ & 1207 & 219 & 48.9 & 49.6 & A \\
\hline HIR 1234+0236 & NGC 4527 & HIPASS J1234+02a & $12: 34: 02$ & $2: 36: 40$ & $1723^{u}$ & $241^{u}$ & $55.1^{u}$ & $56.2^{u}$ & $\mathrm{~V}$ \\
\hline HIR 1234+0332 & UGC 07715 & & $12: 34: 03$ & $3: 32: 42$ & 1101 & 114 & 0.7 & 1.8 & $\mathrm{~V}$ \\
\hline HIR 1234+0212 & NGC 4536 & HIPASS J1234+02b & $12: 34: 34$ & $2: 12: 32$ & $1776^{u}$ & $153^{u}$ & $29.6^{u}$ & $34.6^{u}$ & $\mathrm{~V}$ \\
\hline HIR 1236+0638 & IC 3576 & HIPASS J1236+06 & $12: 36: 35$ & $6: 38: 14$ & 1070 & 71 & 13.0 & 13.4 & A \\
\hline HIR 1236+0306 & UGC 07780 & HIPASS J1236+03 & $12: 36: 40$ & $3: 06: 19$ & 1445 & 141 & 2.9 & 4.1 & $\mathrm{~A}$ \\
\hline HIR 1237+0655 & IC 3591 & HIPASS J1237+06 & 12:37:00 & $6: 55: 52$ & 1626 & 132 & 7.1 & 7.9 & A \\
\hline HIR 1239-0031 & NGC 4592 & HIPASS J1239-00 & $12: 39: 17$ & $-0: 31: 19$ & 1070 & 172 & 162.7 & 98.1 & A \\
\hline HIR 1241+0124 & UGC 07841 & HIPASS J1241+01 & $12: 41: 15$ & $1: 24: 40$ & $1670^{u}$ & $158^{u}$ & $9.4^{u}$ & $7.0^{u}$ & A \\
\hline HIR $1242+0547$ & VCC 1918 & HIPASS J1242+05 & $12: 42: 18$ & 5:47:07 & 983 & 112 & 1.4 & 1.8 & A \\
\hline HIR 1242-0120 & NGC 4629 & HIPASS J1242-01 & $12: 42: 28$ & $-1: 20: 26$ & 1109 & 172 & 22.7 & 22.8 & A \\
\hline HIR $1242+0358$ & NGC 4630 & HIPASS J1242+03 & $12: 42: 32$ & $3: 58: 17$ & 719 & 151 & 4.0 & 5.6 & A \\
\hline HIR 1242-0004 & NGC 4632 & HIPASS J1242-00 & $12: 42: 33$ & $-0: 04: 57$ & $1696^{u}$ & $185^{u}$ & $36.6^{u}$ & $34.1^{u}$ & A \\
\hline HIR $1243+0739$ & VCC 1952 & HIPASS J1243+07 & 12:43:11 & $7: 39: 24$ & 1311 & 86 & 1.9 & 2.4 & A \\
\hline HIR $1243+1132$ & NGC 4647 & $\alpha \alpha$ & $12: 43: 28$ & $11: 32: 37$ & 1394 & 201 & 2.4 & 4.3 & A \\
\hline HIR 1244+0028 & UGC 07911 & HIPASS J1244+00 & $12: 44: 23$ & $0: 28: 19$ & 1178 & 124 & 12.1 & 10.4 & A \\
\hline HIR 1245-0027 & NGC 4666 & HIPASS J1245-00 & $12: 45: 11$ & $-0: 27: 43$ & 1524 & 402 & 75.3 & 78.0 & $\mathrm{~A}$ \\
\hline HIR 1246+0557 & UGC 07943 & HIPASS J1246+05 & $12: 46: 45$ & $5: 57: 37$ & 830 & 141 & 6.2 & 7.6 & A \\
\hline HIR $1247+0420$ & NGC 4688 & HIPASS J1247+04 & $12: 47: 46$ & $4: 20: 33$ & 982 & 73 & 31.1 & 31.8 & A \\
\hline HIR $1247+1058$ & VCC 2062 & $\alpha \alpha$ & $12: 47: 56$ & 10:58:09 & 1140 & 131 & 6.7 & 9.1 & A \\
\hline HIR $1248+0826$ & NGC 4698 & HIPASS J1248+08 & $12: 48: 28$ & $8: 26: 22$ & 995 & 430 & 19.9 & 17.9 & A \\
\hline HIR 1249+0325 & NGC 4701 & HIPASS J1249+03 & 12:49:11 & $3: 25: 10$ & 722 & 178 & 58.2 & 55.7 & A \\
\hline HIR 1249+0519 & NGC 4713 & HIPASS J1250+05 & $12: 49: 57$ & $5: 19: 33$ & 649 & 189 & 47.5 & 46.9 & A \\
\hline HIR $1253+0428$ & NGC 4765 & HIPASS J1253+04 & $12: 53: 11$ & 4:28:09 & 719 & 118 & 13.4 & 19.2 & A \\
\hline HIR 1253+0115 & NGC 4771 & HIPASS J1253+01 & $12: 53: 24$ & 1:15:08 & 1128 & 304 & 9.3 & 9.9 & A \\
\hline HIR $1253+0212$ & NGC 4772 & HIPASS J1253+02 & $12: 53: 29$ & $2: 12: 49$ & 1035 & 43 & 6.9 & 6.8 & A \\
\hline
\end{tabular}


Table 1. continued.

\begin{tabular}{|c|c|c|c|c|c|c|c|c|c|}
\hline Name & Optical ID. & HIPASS ID & RA [hh:mm:ss] & Dec [dd:mm:ss] & $V_{\mathrm{Hel}}{ }^{a}$ & $W_{20}{ }^{b}$ & $S_{\text {int }^{c}}$ & $S_{\text {line }}{ }^{d}$ & det. \\
\hline HIR $1254+0240$ & NGC 4809 & & $12: 54: 51$ & $2: 40: 16$ & 918 & 158 & 10.9 & 11.7 & A \\
\hline HIR $1255+0008$ & UGC 08041 & HIPASS J1255+00 & $12: 55: 12$ & 0:08:12 & 1311 & 205 & 17.0 & 17.9 & $\mathrm{~A}$ \\
\hline HIR $1255+0414$ & NGC 4808 & HIPASS J1255+04b & $12: 55: 50$ & $4: 14: 24$ & 741 & 270 & 81.4 & 60.5 & A \\
\hline HIR 1257+0242 & UGC 08074 & HIPASS J1257+02 & $12: 57: 49$ & $2: 42: 26$ & 918 & 113 & 3.0 & 3.9 & A \\
\hline HIR $1300+0230$ & NGC 4900 & HIPASS J1300+02b & 13:00:37 & $2: 30: 28$ & 943 & 133 & 16.8 & 17.2 & A \\
\hline HIR 1300-0000 & NGC 4904 & HIPASS J1300-00 & $13: 00: 53$ & $-0: 00: 55$ & 1167 & 198 & 8.0 & 9.0 & A \\
\hline HIR 1306+1027 & CGCG 071-109 & HIPASS J1306+10 & $13: 06: 26$ & 10:27:07 & 928 & 65 & 3.1 & 4.4 & A \\
\hline HIR 1311+0530 & UGC 08276 & HIPASS J1312+05 & 13:11:56 & 5:30:11 & 908 & 113 & 2.6 & 3.5 & A \\
\hline HIR 1312+0711 & UGC 08285 & HIPASS J1312+07 & $13: 12: 32$ & 7:11:02 & 890 & 134 & 3.8 & 4.9 & A \\
\hline HIR 1313+1012 & UGC 08298 & HIPASS J1313+10 & $13: 13: 19$ & $10: 12: 16$ & 1152 & 96 & 12.9 & 12.3 & A \\
\hline HIR 1317-0100 & UM 559 & HIPASS J1317-00 & $13: 17: 43$ & $-1: 00: 31$ & 1207 & 111 & 3.9 & 3.4 & A \\
\hline HIR $1320+0524$ & UGC 08382 & HIPASS J1320+05 & $13: 20: 36$ & 5:24:36 & 956 & 119 & 4.6 & 4.3 & A \\
\hline HIR $1320+0947$ & UGC 08385 & HIPASS J1320+09 & $13: 20: 38$ & $9: 47: 51$ & 1115 & 162 & 11.2 & 14.1 & A \\
\hline HIR 1326+0206 & NGC 5147 & HIPASS J1326+02 & $13: 26: 19$ & $2: 06: 48$ & 1087 & 171 & 13.1 & 17.3 & A \\
\hline HIR 1326+0229 & SDSS J132615.73+022729.5 & HIPASS J1326+02B & $13: 26: 29$ & $2: 29: 57$ & 1035 & 110 & 0.8 & 1.0 & $\mathrm{~V}$ \\
\hline HIR $1327+1003$ & UGC 08450 & HIPASS J1327+10 & $13: 27: 08$ & $10: 03: 44$ & 1049 & 103 & 4.4 & 3.2 & A \\
\hline HIR $1328+0219$ & HIPASS J1328+02 & HIPASS J1328+02 & $13: 28: 05$ & $2: 19: 03$ & 1022 & 73 & 7.4 & 3.1 & A \\
\hline HIR $1337+0853$ & NGC 5248 & HIPASS J1337+08 & 13:37:17 & $8: 53: 53$ & 1158 & 288 & 100.1 & 78.0 & A \\
\hline HIR 1337+0739 & UGC 08614 & HIPASS J1337+07 & $13: 37: 27$ & $7: 39: 15$ & 1040 & 175 & 16.0 & 22.9 & A \\
\hline HIR $1338+0826$ & UGC 08629 & HIPASS J1338+08 & $13: 38: 40$ & $8: 26: 50$ & 1022 & 143 & 0.9 & 3.2 & $\mathrm{~V}$ \\
\hline HIR $1348+0356$ & NGC 5300 & HIPASS J1348+03 & $13: 48: 10$ & $3: 56: 31$ & 1170 & 221 & 11.0 & 15.2 & A \\
\hline HIR 1352-0105 & NGC 5334 & HIPASS J1352-01 & $13: 52: 53$ & $-1: 05: 30$ & 1387 & 228 & 24.8 & 26.7 & A \\
\hline HIR $1355+0504$ & NGC 5364 & HIPASS J1356+05 & $13: 55: 41$ & $5: 04: 48$ & 1260 & 291 & 49.4 & 48.8 & A \\
\hline HIR 1401+0247 & No object found. & new & 14:01:04 & $2: 47: 58$ & 1040 & 111 & 3.4 & 2.8 & $\mathrm{~V}$ \\
\hline HIR 1404+0848 & UGC 08995 & HIPASS J1404+08b & 14:04:49 & 8:48:43 & 1234 & 182 & 9.2 & 7.5 & A \\
\hline HIR 1411-0109 & NGC 5496 & HIPASS J1411-01 & $14: 11: 38$ & $-1: 09: 18$ & 1541 & 267 & 67.7 & 66.2 & A \\
\hline HIR 1416+0350 & HIPASS J1416+03 & HIPASS J1416+03 & 14:16:59 & $3: 50: 21$ & 1470 & 116 & 7.0 & 8.8 & A \\
\hline HIR 1417-0130 & 2dFGRS N275Z229 & HIPASS J1417-01 & 14:17:14 & $-1: 30: 07$ & 1551 & 88 & 2.2 & 2.4 & A \\
\hline HIR 1417+0651 & No object found & new & $14: 17: 45$ & $6: 51: 08$ & 1167 & 92 & 2.3 & 2.4 & V \\
\hline HIR 1419+0922 & UGC 09169 & HIPASS J1419+09 & $14: 19: 44$ & $9: 22: 36$ & 1280 & 165 & 20.3 & 23.1 & $\mathrm{~A}$ \\
\hline HIR $1420+0358$ & NGC 5569 & & $14: 20: 29$ & $3: 58: 41$ & $1750^{u}$ & $135^{u}$ & $7.8^{u}$ & $9.0^{u}$ & A \\
\hline HIR $1420+0834$ & SDSS J142044.53+083735.8 & HIPASS J1420+08 & $14: 20: 48$ & $8: 34: 55$ & 1286 & 130 & 2.5 & 3.2 & V \\
\hline HIR $1421+0326$ & NGC 5577 & HIPASS J1421+03 & $14: 21: 28$ & $3: 26: 51$ & 1485 & 275 & 9.0 & 8.4 & $\mathrm{~A}$ \\
\hline HIR 1422-0022 & NGC 5584 & HIPASS J1422-00 & $14: 22: 25$ & $-0: 22: 57$ & 1657 & 223 & 30.8 & 31.0 & A \\
\hline HIR 1423+0143 & UGC 09215 & HIPASS J1423+01 & $14: 23: 29$ & $1: 43: 21$ & 1383 & 239 & 20.7 & 22.1 & A \\
\hline HIR 1424+0820 & UGC 09225 & HIPASS J1424+08 & $14: 24: 22$ & 8:20:17 & 1247 & 130 & 4.9 & 5.6 & A \\
\hline HIR $1427+0842$ & UGC 09249 & HIPASS J1427+08 & 14:27:02 & 8:42:16 & 1365 & 148 & 9.8 & 9.7 & A \\
\hline HIR 1429-0000 & UGC 09299 & HIPASS J1429-00 & $14: 29: 34$ & $-0: 00: 24$ & 1535 & 213 & 50.8 & 46.3 & A \\
\hline HIR 1430+0717 & NGC 5645 & HIPASS J1430+07 & $14: 30: 39$ & 7:17:03 & 1365 & 195 & 19.3 & 17.3 & A \\
\hline HIR $1431+0257$ & IC 1024 & HIPASS J1431+03 & 14:31:21 & $2: 57: 38$ & 1445 & 215 & 8.6 & 10.9 & $\mathrm{~V}$ \\
\hline HIR 1432+0954 & NGC 5669 & HIPASS J1432+09 & $14: 32: 41$ & $9: 54: 10$ & 1365 & 211 & 31.8 & 41.7 & A \\
\hline HIR 1432+0257 & CGCG 047-085 & & $14: 32: 42$ & $2: 57: 51$ & 1537 & 146 & 4.1 & 5.4 & V \\
\hline HIR 1432+0016 & UGC 09348 & & $14: 32: 55$ & $0: 16: 36$ & 1657 & 198 & 5.5 & 5.2 & V \\
\hline HIR 1433+0426 & NGC 5668 & HIPASS J1433+04 & $14: 33: 29$ & $4: 26: 57$ & 1577 & 124 & 50.7 & 50.1 & A \\
\hline HIR $1435+0517$ & UGC 09385 & HIPASS J1435+05 & $14: 35: 22$ & $5: 17: 30$ & 1635 & 106 & 7.4 & 7.8 & A \\
\hline HIR 1437+0217 & NGC 5690 & HIPASS J1437+02 & $14: 37: 40$ & $2: 17: 30$ & $1710^{u}$ & $193^{u}$ & $10.2^{u}$ & $13.0^{u}$ & A \\
\hline HIR 1439+0259 & UGC 09432 & HIPASS J1439+02 & $14: 39: 06$ & $2: 59: 08$ & 1564 & 110 & 7.4 & 6.4 & A \\
\hline HIR 1439+0521 & NGC 5701 & HIPASS J1439+05 & $14: 39: 10$ & $5: 21: 44$ & 1499 & 139 & 77.2 & 57.4 & A \\
\hline HIR 1439-0040 & NGC 5705 & HIPASS J1439-00 & 14:39:45 & $-0: 40: 20$ & $1736^{u}$ & $136^{u}$ & $18.2^{u}$ & $19.2^{u}$ & A \\
\hline HIR 1440-0026 & NGC 5719 & HIPASS J1440-00 & 14:40:43 & $-0: 26: 53$ & 1643 & 247 & 36.9 & 31.2 & A \\
\hline HIR $1440+0210$ & NGC 5725 & HIPASS J1440+02 & $14: 40: 46$ & $2: 10: 53$ & 1617 & 179 & 1.8 & 3.4 & V \\
\hline HIR 1443+0451 & IC 1048 & HIPASS J1443+04 & $14: 43: 02$ & $4: 51: 25$ & $1670^{u}$ & $307^{u}$ & $19.0^{u}$ & $18.2^{u}$ & V \\
\hline HIR 1444+0142 & NGC 5740 & HIPASS J1444+01 & $14: 44: 25$ & $1: 42: 53$ & 1556 & 334 & 30.4 & 23.8 & A \\
\hline HIR 1445+0751 & UGC 09500 & HIPASS J1445+07 & $14: 45: 18$ & 7:51:59 & 1682 & 56 & 9.7 & 9.4 & A \\
\hline HIR 1446+1011 & No object found & new & $14: 46: 27$ & 10:11:55 & 969 & 154 & 3.5 & 3.9 & V \\
\hline HIR 1453+0333 & NGC 5774 & HIPASS J1453+03 & $14: 53: 45$ & $3: 33: 13$ & $1723^{u}$ & $308^{u}$ & $89.4^{u}$ & $84.9^{u}$ & A \\
\hline HIR 1458-0104 & NGC 5792 & HIPASS J1458-01 & 14:58:30 & $-1: 04: 54$ & $1740^{u}$ & $92^{u}$ & $16.4^{u}$ & $16.9^{u}$ & A \\
\hline HIR 1458+0646 & KUG 1456+069 & HIPASS J1458+06 & $14: 58: 35$ & $6: 46: 30$ & 1681 & 138 & 6.2 & 4.6 & A \\
\hline HIR $1500+0155$ & NGC 5806 & HIPASS J1500+01 & 15:00:00 & $1: 55: 55$ & 1352 & 322 & 9.1 & 7.4 & V \\
\hline HIR 1504-0052 & UGC 09682 & HIPASS J1504-00 & $15: 04: 20$ & $-0: 52: 29$ & $1776^{u}$ & $101^{u}$ & $3.9^{u}$ & $3.5^{u}$ & A \\
\hline HIR 1515+0603 & No object found & new & $15: 15: 02$ & $6: 03: 22$ & 1696 & 103 & 2.2 & 3.1 & V \\
\hline HIR $1521+0505$ & NGC 5921 & HIPASS J1521+05 & $15: 21: 56$ & 5:05:01 & 1472 & 189 & 35.2 & 31.9 & A \\
\hline HIR 1537+0558 & NGC 5964 & HIPASS J1537+05 & $15: 37: 35$ & $5: 58: 48$ & 1446 & 212 & 36.5 & 38.6 & A \\
\hline HIR 1546+0654 & UGC 10023 & HIPASS J1546+06 & $15: 46: 05$ & $6: 54: 55$ & 1407 & 170 & 5.5 & 5.2 & A \\
\hline HIR 1605+0832 & CGCG 079-046 & HIPASS J1606+08 & $16: 05: 40$ & 8:32:03 & 1379 & 153 & 4.3 & 4.7 & $\mathrm{~V}$ \\
\hline
\end{tabular}


Table 1. continued.

\begin{tabular}{|c|c|c|c|c|c|c|c|c|c|}
\hline Name & Optical ID. & HIPASS ID & RA [hh:mm:ss] & Dec [dd:mm:ss] & $V_{\mathrm{Hel}}^{a}$ & $W_{20}{ }^{b}$ & $S_{\mathrm{int}^{c}}^{c}$ & $S_{\text {line }} d$ & det. \\
\hline HIR 1608+0733 & IC 1197 & HIPASS J1608+07 & 16:08:15 & $7: 33: 15$ & 1360 & 221 & 13.5 & 11.4 & A \\
\hline HIR 1609-0006 & UGC 10229 & HIPASS J1609-00 & 16:09:42 & $-0: 06: 08$ & 1491 & 129 & 9.1 & 8.1 & A \\
\hline HIR 1618+0725 & NGC 6106 & HIPASS J1618+07 & $16: 18: 45$ & $7: 25: 10$ & 1445 & 253 & 19.7 & 20.7 & A \\
\hline HIR 1619+0142 & CGCG 024-001 & HIPASS J1619+01a & 16:19:19 & 1:42:02 & 1497 & 165 & 6.8 & 9.5 & A \\
\hline HIR 1656+0800 & HIPASS J1656+08 & HIPASS J1656+08 & $16: 56: 39$ & $8: 00: 53$ & 1471 & 113 & 3.0 & 4.3 & A \\
\hline HIR 1728+0725 & UGC 10862 & HIPASS J1728+07 & $17: 28: 07$ & $7: 25: 35$ & 1685 & 150 & 13.1 & 15.7 & A \\
\hline HIR 1732+0705 & NGC 6384 & HIPASS J1732+07 & $17: 32: 26$ & $7: 05: 45$ & $1683^{u}$ & $313^{u}$ & $42.6^{u}$ & $50.5^{u}$ & A \\
\hline
\end{tabular}

Notes. ${ }^{(a)}$ Units of $V_{\mathrm{Hel}}$ are given in $\left[\mathrm{km} \mathrm{s}^{-1}\right] .{ }^{(b)}$ Units of $W_{20}$ are given in $\left[\mathrm{km} \mathrm{s}^{-1}\right] .{ }^{(c)}$ Units of $S_{\text {int }}$ are given in $\left[\mathrm{Jy} \mathrm{km} \mathrm{s}{ }^{-1}\right] .{ }^{(d)}$ Units of $S_{\text {line }}$ are given in $\left[\mathrm{Jy} \mathrm{km} \mathrm{s}^{-1}\right] .{ }^{\left({ }^{\prime}\right)}$ The flux and line-width values are underestimated as the object is at the edge of the processed bandwidth.

is most likely the H I component of CGCG 014-054 because of the good correspondence in position and velocity.

HIR 1401+0247: there is no known galaxy at the relevant redshift, however when looking at the DSS image, there is the massive galaxy cluster Abell 1835 at redshift 0.253 centered at $\mathrm{RA}=14: 01: 02.0$ and $\mathrm{Dec}=02: 51: 32$, coincident with the peak of the apparent Hi contours. An association with an $\mathrm{H}_{\mathrm{I}}$ signal at the cluster redshift is clearly out of the question, while applying the cluster redshift to the detected spectral feature would imply a rest frequency of $1773.6 \mathrm{MHz}$, where no known transition occurs. For comparison, some known radio frequency transitions are $\mathrm{OH} 1720.53 \mathrm{MHz}, \mathrm{H}_{2} \mathrm{CO} 4829.66 \mathrm{MHz}$, and $\mathrm{CH}_{3} \mathrm{OH} 6668.52 \mathrm{MHz}$. The cluster is known to act as a gravitational lens (Sand et al. 2005), although current attempts to determine the redshift of lensed features have not been successful. While unlikely, the detected feature might correspond to $\mathrm{H}_{2} \mathrm{CO}$ at $z=2.4$, or $\mathrm{CH}_{3} \mathrm{OH}$ at $z=3.7$.

HIR 1417+0651: when looking at the DSS image at the location of this object, two small galaxies can be recognised at the peak of the contours. One is CGCG 046-087 which is irrelevant because of the radial velocity of $7559 \mathrm{~km} \mathrm{~s}^{-1}$. The other object is a GALEX source at RA $=14: 17: 50.7$ and Dec $=06: 50: 22$ without any redshift information.

HIR 1446+1011: the DSS image shows a galaxy at the peak of the Hi contours. However, this is CGCG 076-029 at almost $16000 \mathrm{~km} \mathrm{~s}^{-1}$. Beyond this, there is no sign of an optical source that can be easily linked to HIR $1446+0011$.

HIR 1515+0603: there is a very small and diffuse SDSS object at $\mathrm{RA}=15: 14: 57.23$ and $\mathrm{Dec}=06: 06: 03.10$. Although there is no redshift information about this object, based on the visual appearance a connection with HIR $1515+0603$ is possible, but not very likely.

\subsection{Flux densities}

The total flux densities have been determined for all detections using two independent methods. The line-widths and the integrated line-strengths have first been determined by integrating the brightness over the full velocity width of an object along the single spectrum that contains the overall peak brightness. We assume that all sources are unresolved and fully contained within the 15.5 arcmin beam. In a second approach the total flux has been determined from the integrated moment maps. Moment maps are created by collapsing the cube in the direction of the velocity axis over the full line width. The visualisation package KARMA (Gooch 1996) has been used to integrate the flux. The radial profile of an object can be plotted, including a fit to the data points within a user defined circle. This circle was chosen to completely enclose the object, including any possible extended emission. The integrated flux densities have to be corrected for the beam integral to convert from Jy Beam ${ }^{-1} \mathrm{~km} \mathrm{~s}^{-1}$ to Jy $\mathrm{km} \mathrm{s}^{-1}$. An integrated flux density is determined by simply adding the pixels values, and a fitted flux density is determined by fitting a Gaussian to the radial profile. Since we aim to be sensitive to extended features that do not necessarily have a Gaussian profile, we use the pixel integral for our flux density determinations rather than a Gaussian fit.

When integrating the line strength of a single spectrum, not all the flux is measured if the source is resolved by the beam, or if there are extended emission features like filaments. The flux densities are plotted and compared in Fig. 3 where in the left panel the total flux obtained from the integrated pixel values is plotted as function of the single pixel integrated line strength. The dotted line goes through the origin and indicates where the fluxes are equal. Only those sources are plotted that are completely covered by our data cubes in both the spectral and spatial directions. The measured fluxes match the dotted line very well, meaning that there is typically no large discrepancy between the two different methods. The ratio of the fluxes is plotted on a logarithmic scale in the right panel of Fig. 3, the dotted line indicating again where the fluxes are equal. The mean of the ratios is 0.99 , with a standard deviation of 0.28 . The dashed line in the right panel of Fig. 3 represents the median of the flux ratios, which is 0.96 . As both the mean and median values are close to one, there is generally very good agreement between the fluxes. For large flux values above $\sim 50 \mathrm{Jy} \mathrm{km} \mathrm{s}^{-1}$ the fluxes derived by integrating the individual pixel values are typically larger by 20 to $50 \%$. This is due to the fact that these are typically large and extended sources that are resolved by the beam. At low flux levels, there are a number of sources for which the integrated linestrength exceeds the integrated flux of the moment map by more than a factor of two. Since the line-strength of the peak spectrum provides a lower limit to the true integral, there must be a residual artefact in either the spectrum or the moment map. The spectra and moment maps of these sources were inspected and either the spectral bandpass appears slightly elevated, or there are negative residuals in the moment maps which influence the flux estimates.

Another important comparison to make is of our measured fluxes with those obtained in the first HIPASS product. In Fig. 4 fluxes derived from the reprocessed HIPASS data are compared with fluxes in the HIPASS catalogue (Meyer et al. 2004; Wong et al. 2006). Again, only those sources are plotted that are completely covered by the data cubes. The left panel shows the reprocessed fluxes as function of original fluxes, while the right panel shows the ratio. The dashed line indicates the median of the flux ratios which is 1.10 . The mean of the ratios is 1.31 with a standard deviation of 1.41 . In this case the median estimate gives a better representation of the general trend instead of the mean, as the effect of strong outliers is suppressed. 

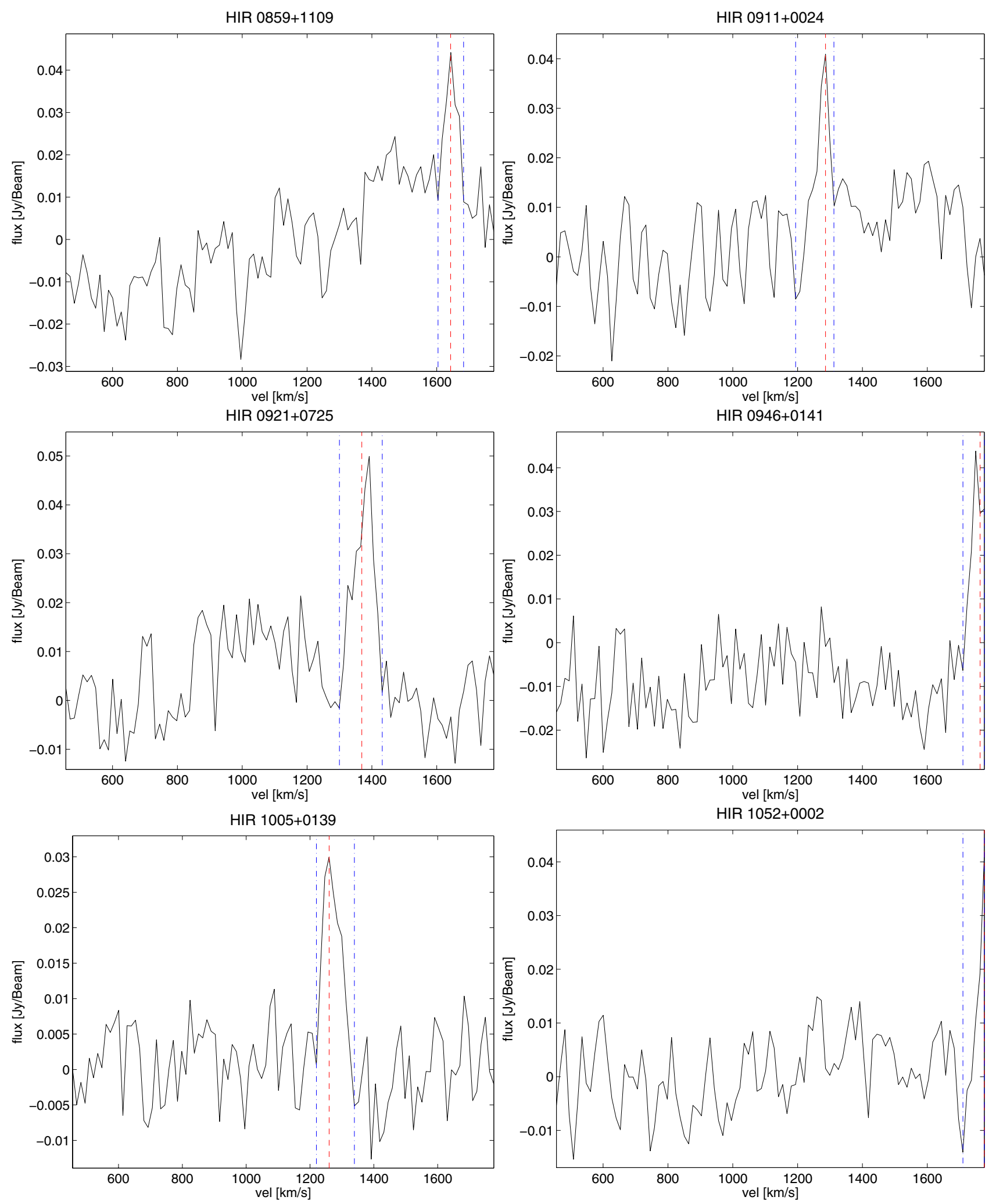

Fig. 2. Spectra of new $\mathrm{H}_{\mathrm{I}}$ detections obtained from reprocessed HIPASS data. The radial velocity of each object is indicated by the red dashed vertical, the velocity width is indicated by the blue dash-dotted line.

The $\sim 10 \%$ excess in flux in the published HIPASS product over that in our reprocessed result may reflect the variation in effective beam size with signal-to-noise ratio that is a consequence of median gridding, as discussed at some length in Barnes et al. (2001). Exactly the same data has been used in both processing methods, so another effect that may contribute to the difference in flux is how the bandpass is determined. The gridding of the data has been done in a similar fashion as for the original HIPASS product, so any differences are caused in the pre-gridding. For several sources the individual spectra in both the original and the re-reduced HIPASS product have been compared. Despite the difference in flux, the spectra look 
A. Popping and R. Braun: Diffuse neutral hydrogen in the H I Parkes All Sky Survey
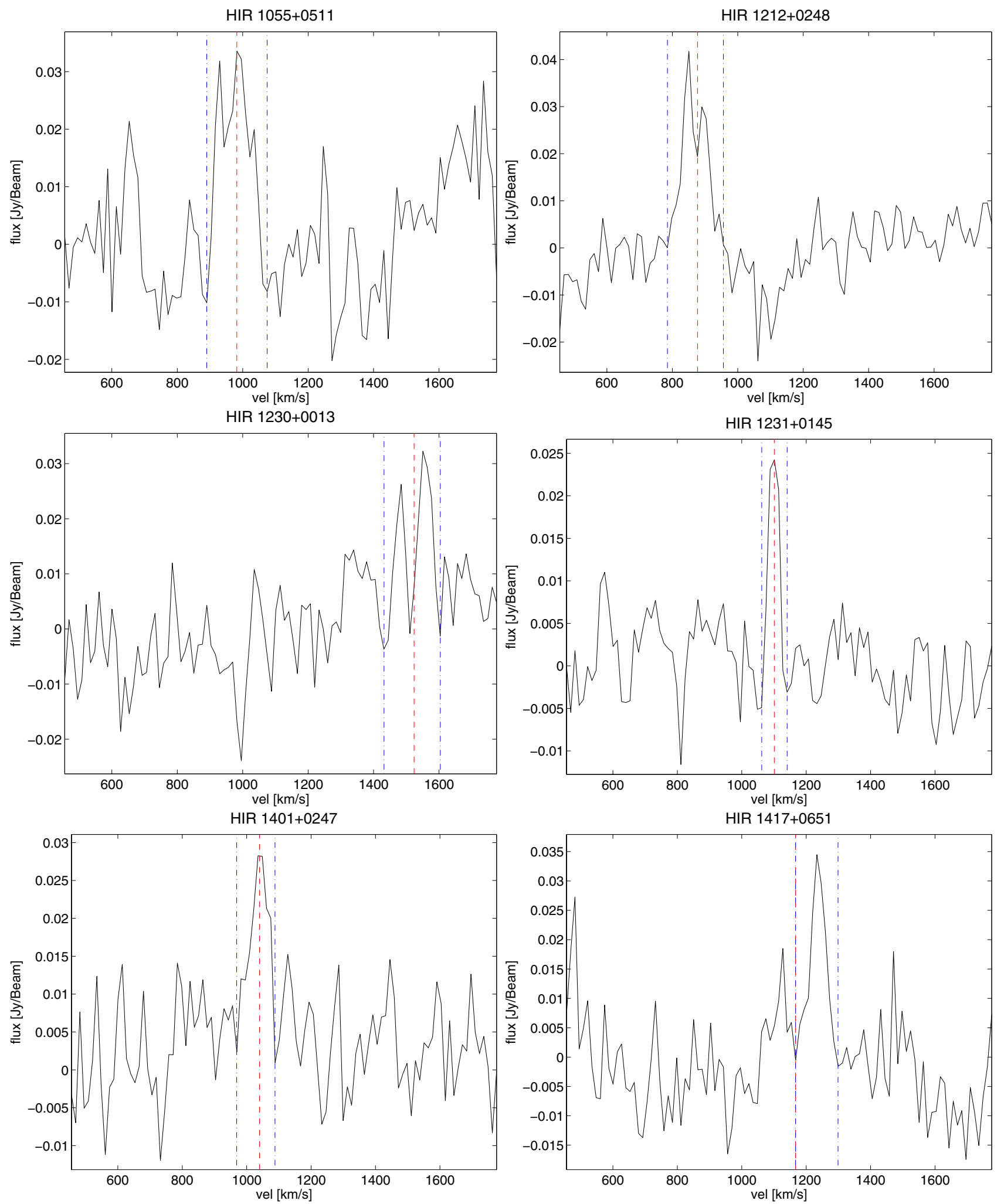

Fig. 2. continued

very similar. A difference in the fit to the bandpass can enhance the whole spectrum slightly, without significantly affecting the shape.

\subsection{Companions}

Moment maps have been generated for all detected objects by integrating over their velocity widths. Initially, maps of 2 by
2 degrees in size are generated, to completely cover the detection itself, including the nearby environment. All moment maps were inspected by eye for diffuse emission features. For objects that showed tentative signs of filaments or companions, another moment map was generated of 5 by 5 degrees in size. These moment maps were inspected in detail, by searching for local peaks in the spatial domain as well as possible line features in the spectrum at the relevant velocity. 

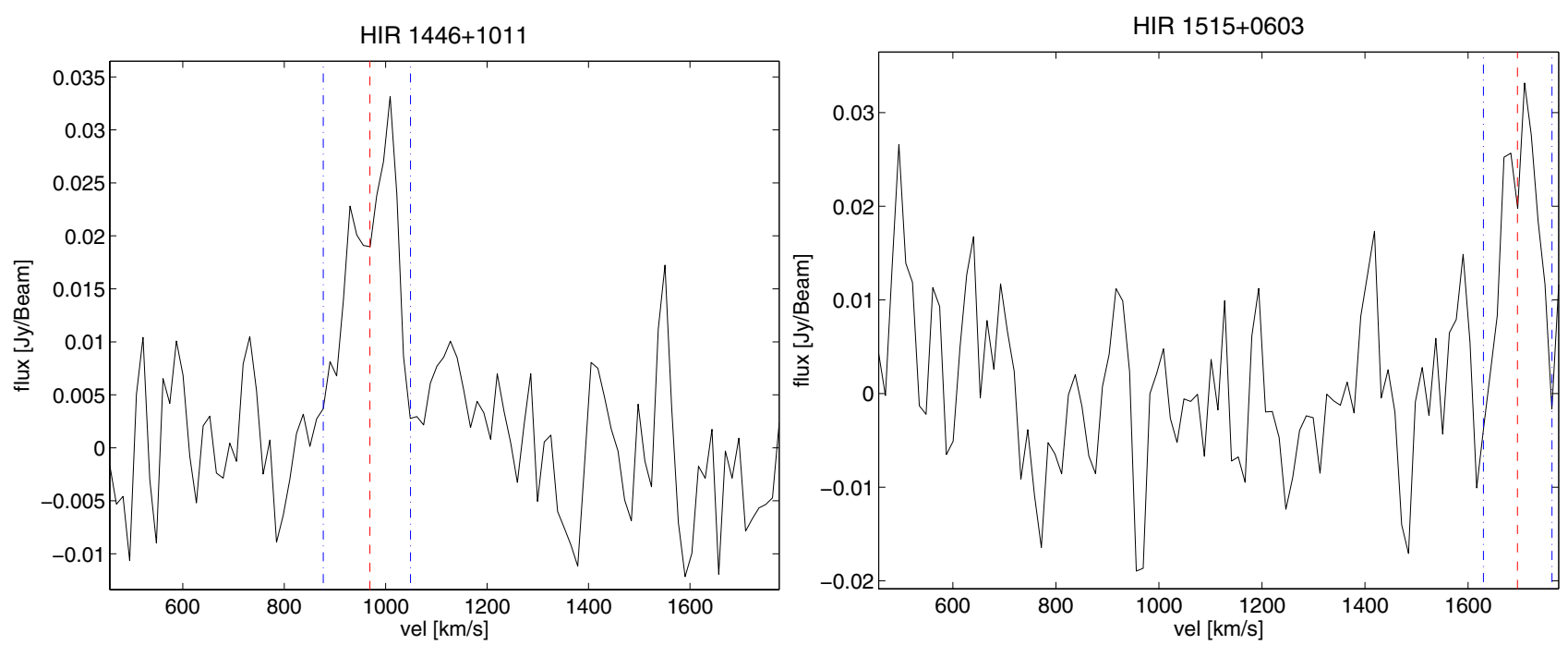

Fig. 2. continued.
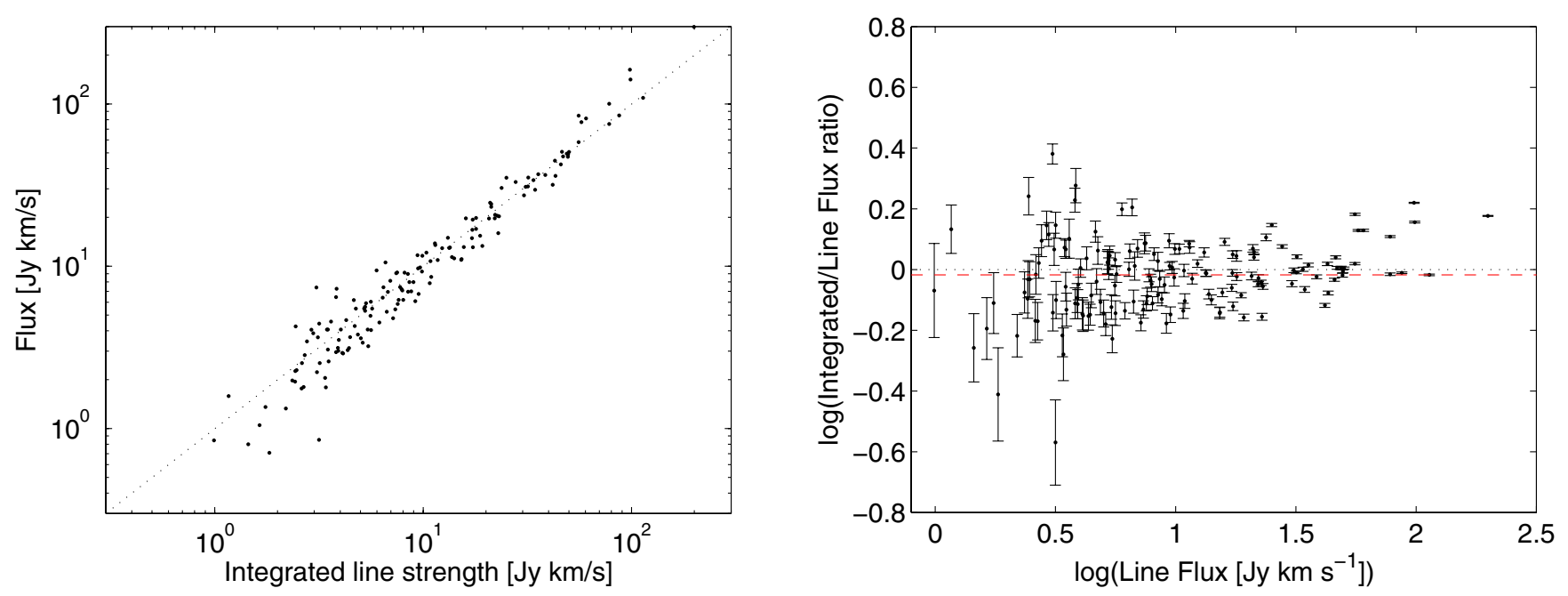

Fig. 3. Left panel: flux obtained from the moment maps is plotted as function of the line strength, the dotted line indicates where the two flux measurements are equivalent. Right panel: ratio of integrated flux and line-strength is plotted as function of line-strength on a logarithmic scale. The dotted line indicates where the ratio is one and the fluxes are equivalent. The dashed line shows the median of the ratios.
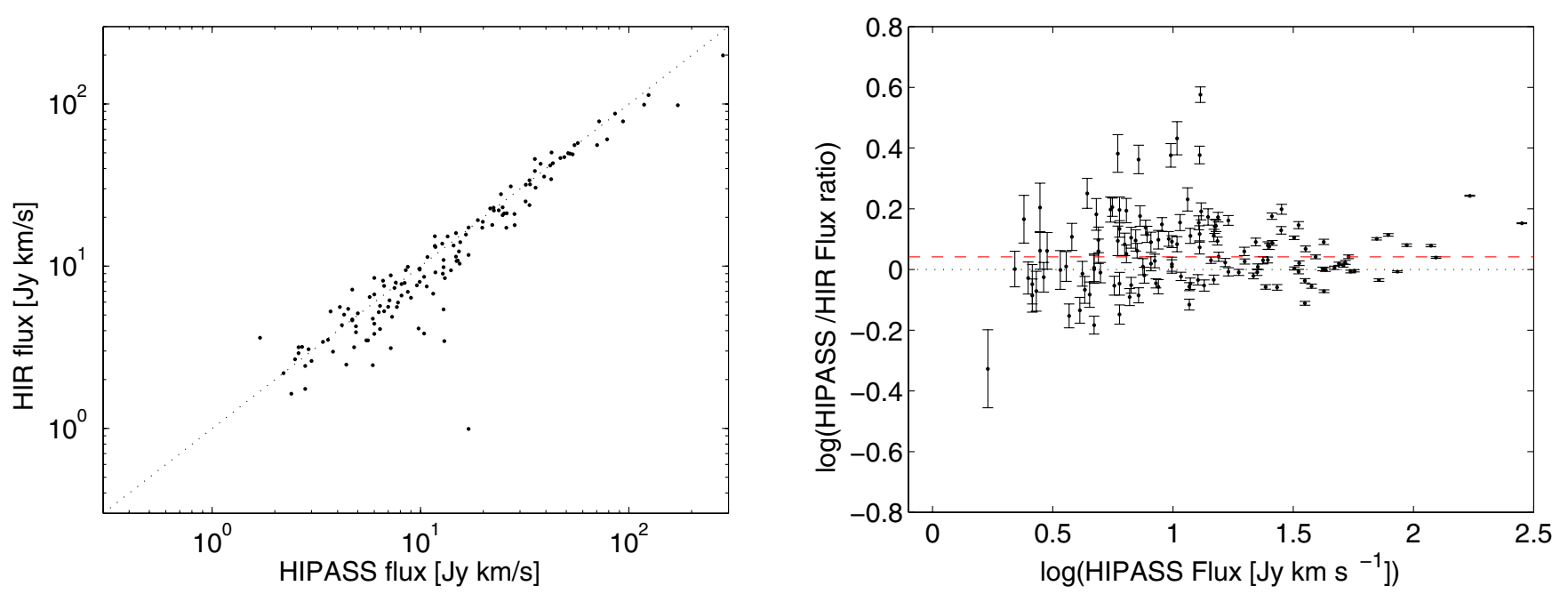

Fig. 4. Left panel: the integrated line strength of the reprocessed HIPASS data (HIR) is plotted as function of the original HIPASS fluxes. Right panel: ratio of the first and reprocessed HIPASS product is plotted against the original HIPASS flux. The dashed line indicates where the fluxes are equal, while the dashed line represents the median of the ratios. On average there is a small excess in the flux values of the original HIPASS product. 
A. Popping and R. Braun: Diffuse neutral hydrogen in the H I Parkes All Sky Survey
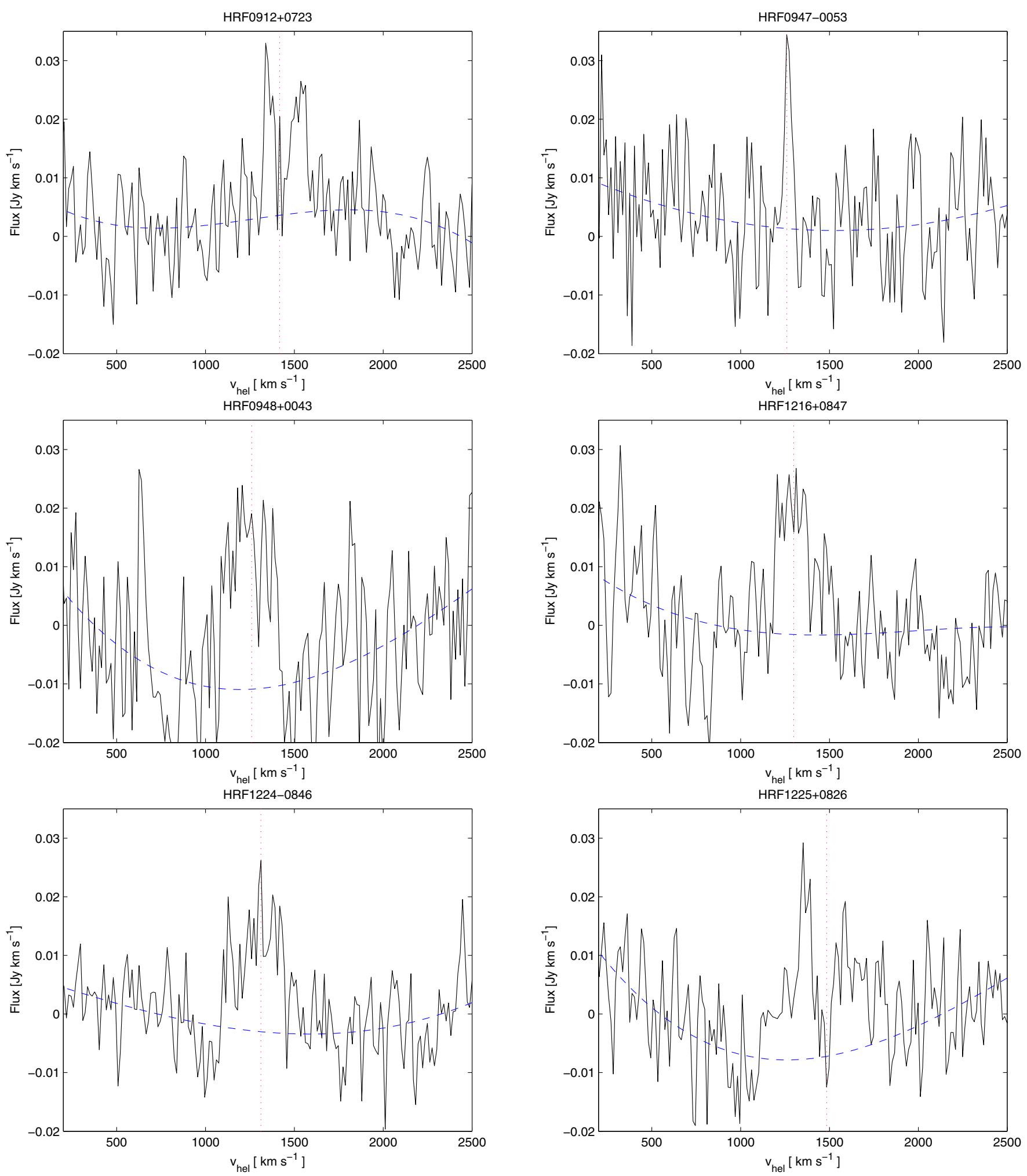

Fig. 5. Spectra of $\mathrm{H}_{\mathrm{I}}$ features that have been detected in the vicinity of other objects, by inspecting the moment maps. A third order polynomial is fit to the bandpass and is indicated by the dashed line. The vertical dotted line represents the radial velocity of the detected feature.

Several faint features have been marginally detected, that have not emerged from our earlier source finding procedure. These detections appear very interesting but would need further confirmation to make them robust. They are usually very faint, but some of them have very broad line-widths. None of the features have an optical counterpart, therefore the origin of the features is not straightforward. All of these tentative detections were found by visually inspecting the moment maps around bright sources. As none of them passed the criteria of previous source finding algorithms, it is very likely that a significant number of comparable features are still present in the data. We will discuss the detailed properties of all features below. The relevant spectra are shown in Fig. 5. A third order polynomial was fit to the spectrum, excluding the line itself and galactic emission, to correct 

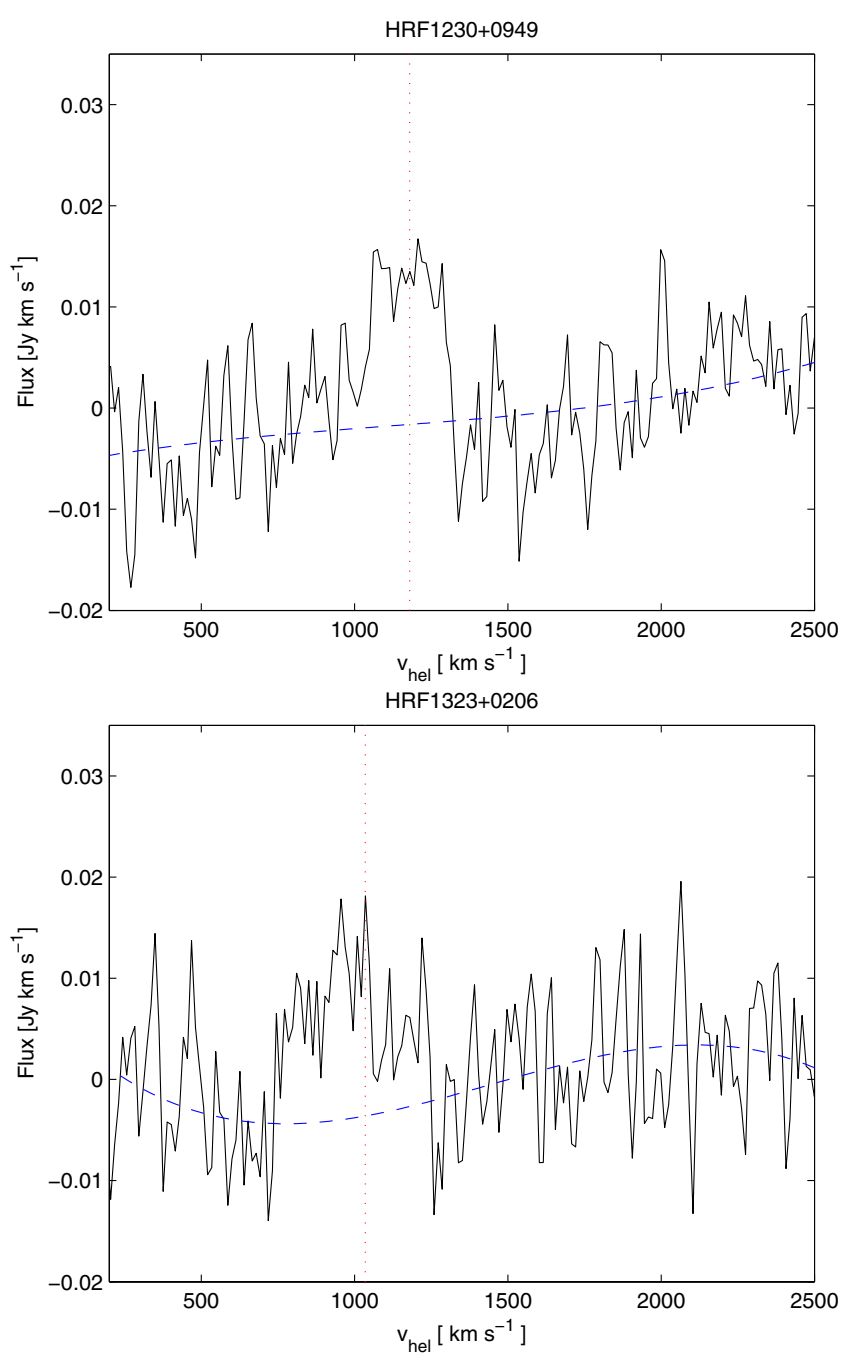

Fig. 5. continued.

for bandpass instabilities at these very low flux values. We will leave statements about the possible origin of these features to the discussion.

HIRF 0912+0723: the position of this feature, at RA = 09:12:55 and Dec $=07: 23: 34$, is close to UGC 4781 and NGC 2777 and it has a comparable radial velocity to these galaxies of $1458 \mathrm{~km} \mathrm{~s}^{-1}$. The spectrum has two peaks, that are separated by $140 \mathrm{~km} \mathrm{~s}^{-1}$. The two peaks are reminiscent of a double horned profile, although they may simply be due to two unrelated structures within the telescope beam. A direct relation to either of the two cataloged galaxies is not obvious, as the line is quite broad and has a very different character. The total line integral at the indicated position is $3.9 \mathrm{Jy} \mathrm{km} \mathrm{s}^{-1}$, after correction of the spectral baseline. With an integrated signal-to-noise of $\sim 7$, this detection has moderately high significance.

HIRF 0947-0053: this feature with RA = 09:47:11 and Dec $=-00: 53: 05$ is near the optical source SDSS J094446.23004118.2 , in the same field as the previous detection. In contrast to HIRF $0948+0043$, this detection is relatively narrow, with a $W_{20}$ value of $77 \mathrm{~km} \mathrm{~s}^{-1}$ and it has one peak with a maximum brightness of $\sim 33 \mathrm{mJy}^{\text {beam }}{ }^{-1}$. The integrated line-strength is only $1.3 \mathrm{Jy} \mathrm{km} \mathrm{s}^{-1}$, yielding a marginally significant signal-tonoise of 5 .

HIRF 0948+0043: this feature is located at RA $=09: 48: 32$ and Dec $=00: 43: 23$, offset by several degrees from NGC 3044
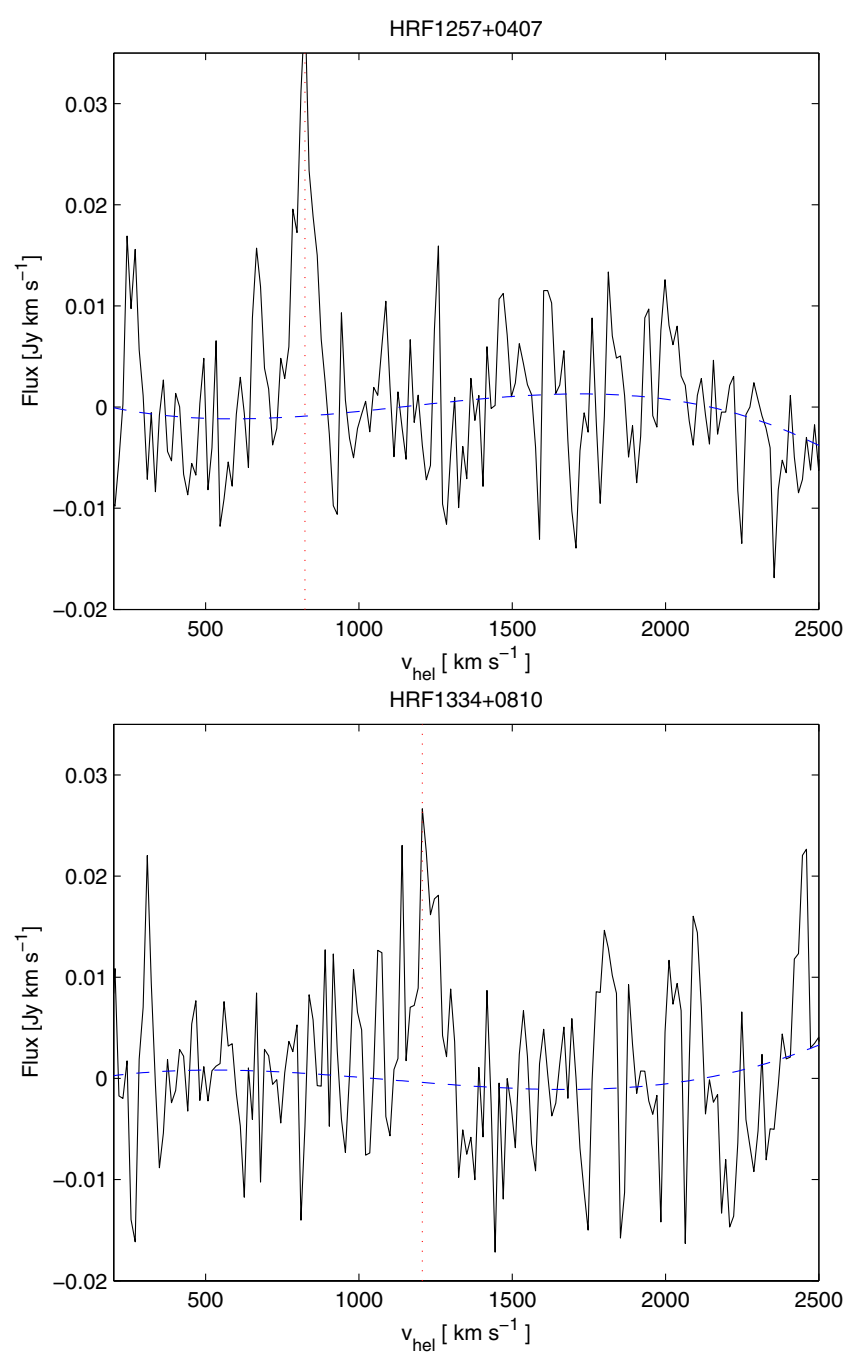

which has a consistent radial velocity of $1289 \mathrm{~km} \mathrm{~s}^{-1}$. This is a very broad profile with a $W_{20}$ value of $\sim 320 \mathrm{~km} \mathrm{~s}^{-1}$. Although the brightness in each channel is only about $10 \mathrm{mJy} \mathrm{Beam}^{-1}$, this brightness is present over 25 channels, which yields a high significance. The line-integral without any further smoothing is $7.6 \mathrm{Jy} \mathrm{km} \mathrm{s}{ }^{-1}$, which corresponds to a signal to noise of 13. Intriguingly, there appear to be additional filamentary features in the field of this object. Similar broad line profiles can be recognised at several positions along the filament, albeit with low signal-to-noise. A DSS image of the region around HIRF $0948+0043$ is shown in Fig. 6, which is indicated by the letter $\mathrm{C}$ in this image. The main galaxy in the top left of the plot is NGC 3044 which has extended emission. Letters B-D are assigned to regions in the environment showing emission. The line profiles are shown in Fig. 7, although the fluxes in all the companions are very low, they all have a line-width that is very comparable to NGC 3044. It appears as if these denser regions form a more extended filament. In Lee \& Irwin (1997) a high resolution H i image of NGC 3044 obtained with the VLA is presented. Although the brightness sensitivity of these observations is much lower than the Parkes data, at column density levels of a few times $N_{\mathrm{HI}} \sim 10^{20} \mathrm{~cm}^{-2}$ small companions can be seen in the same direction as the companions we detect.

HIRF 1216+847: at RA $=12: 16: 45$ and Dec $=08: 47: 22$ this detection has a radial velocity of $1299 \mathrm{~km} \mathrm{~s}^{-1}$ and 


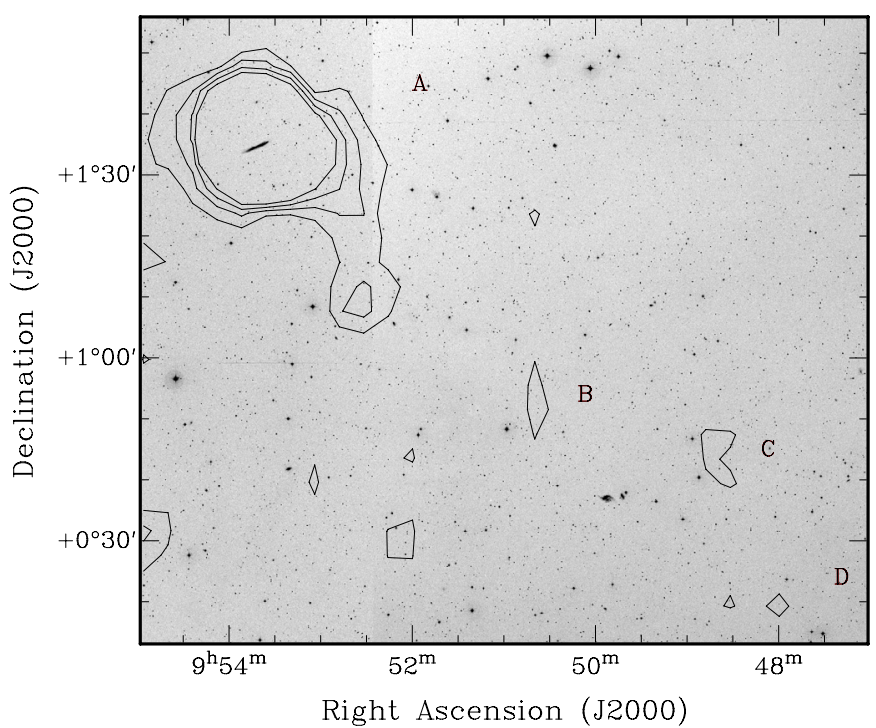

Fig. 6. Second generation DSS image with $\mathrm{H}_{\mathrm{I}}$ contours at $2-5 \mathrm{Jy}_{\text {beam }}^{-1} \mathrm{~km} \mathrm{~s}^{-1}$. The large galaxy is NGC 3044, three companions are identified with very similar line-widths. The line profile of each object is shown in Fig. 7.

$W_{20}=199 \mathrm{~km} \mathrm{~s}^{-1}$. Although the peak brightness is not very substantial; 20 mJy beam ${ }^{-1}$ over the full line width, the integrated flux of $4.3 \mathrm{Jy} \mathrm{km} \mathrm{s}^{-1}$ corresponds to a signal-to-noise of 9 .

HIRF 1224+0846: with an RA of 12:24:52 and Dec of 08:46:25, this detection is within half a degree of NGC 4316. The radial velocity of $1312 \mathrm{~km} \mathrm{~s}^{-1}$ matches that of NGC 4316 fairly well. Of particular note is the very broad line-width of approximately $400 \mathrm{~km} \mathrm{~s}^{-1}$. While the peak brightness is modest; only $24 \mathrm{mJy} \mathrm{beam}^{-1}$ it extends quite uniformly across the entire line-width. This yields an integrated flux of $5.3 \mathrm{Jy} \mathrm{km} \mathrm{s}{ }^{-1}$ corresponding to a signal-to-noise of 9.

HRIF 1225+0826: located at RA $=12: 25: 40$ and Dec $=$ 08:26:16, this intriguing feature's properties are very sensitive to the method of baseline definition. The overlaid baseline fit results in a central radial velocity of $1484 \mathrm{~km} \mathrm{~s}^{-1}$, an integrated flux of $8.4 \mathrm{Jy} \mathrm{km} \mathrm{s}^{-1}$, an extremely broad line-width of about $700 \mathrm{~km} \mathrm{~s}^{-1}$ at $20 \%$ of the peak flux and a signal-to-noise of 10 . However, other spectral baselines would severely diminish the apparent line-width, flux and overall significance of this feature. A slightly enhanced baseline fit would also cut the source in half, therefore potentially this detection could also consist of two different sources with a different spectral position within the telescope beam. Confirming observations will be necessary to establish it's reliability.

HRIF 1230+0949: this is a very similar detection to several of those discussed previously, with a low peak brightness, but a very broad line-width. This objects is located at RA $=12: 30: 24$ and $\operatorname{Dec}=09: 49: 45$ with a radial velocity of $1180 \mathrm{~km} \mathrm{~s}^{-1}$. The integrated flux of $3.7 \mathrm{Jy} \mathrm{km} \mathrm{s}^{-1}$ over a line-width $W 2_{20}$ of $280 \mathrm{~km} \mathrm{~s}^{-1}$, yield a signal-to-noise of 7 .

HIRF 1257+0407: this detection at RA $=12: 57: 20$ and Dec $=04: 07: 59$ is in the direct environment of NGC 4804 with a radial velocity of $824 \mathrm{~km} \mathrm{~s}^{-1}$ that is comparable to this galaxy. The peak flux of $35 \mathrm{mJy}^{\text {beam s}} \mathrm{s}^{-1}$ is relatively strong, compared to the other detections listed here. The line-width at $20 \%$ of the peak is $102 \mathrm{~km} \mathrm{~s}^{-1}$ and the integrated flux of $2.4 \mathrm{Jy} \mathrm{km} \mathrm{s}^{-1}$ has a signal-to-noise of 7 .

HIRF 1323+0206: this detection is at RA = 13:23:17 and Dec $=02: 06: 06$, with a radial velocity of $980 \mathrm{~km} \mathrm{~s}^{-1}$. This is another example of a very faint source with a very broad line width of more than $500 \mathrm{~km} \mathrm{~s}^{-1}$. The brightness in each individual channel barely exceeds the $1 \sigma$ level. The line-width at $20 \%$ of the peak is $505 \mathrm{~km} \mathrm{~s}^{-1}$ and the integrated line strength is $5.2 \mathrm{Jy} \mathrm{km} \mathrm{s}^{-1}$, which corresponds to a signal-to-noise of 7 .

HIRF 1334+0810: this detection at RA $=13: 34: 21$ and Dec $=08: 10: 14$ is only marginal. The peak brightness is $\sim 25 \mathrm{mJy}$ beam $^{-1}$ and the integrated flux is $2.2 \mathrm{Jy} \mathrm{km} \mathrm{s}^{-1}$ and has a signal to noise of only 6.5 when taking into account the $W_{20}$ of $110 \mathrm{~km} \mathrm{~s}^{-1}$. Interesting however is that this feature is about half a degree south of an extended chain of galaxies that is connected to NGC 5248 in the data. Although these galaxies individually could not be resolved in the HIPASS data, optical images from DSS can reveal UGC 8575 and CGCG 073-036. Both these galaxies and HIRF $1324+0810$ have a similar radial velocity of $\sim 1200 \mathrm{~km} \mathrm{~s}^{-1}$ and a narrow line profile that is completely embedded in the profile of NGC 5248. This diffuse detection seems to be connected to the filament of galaxies, although the connecting bridge is very faint.

\subsection{Completeness and robustness}

Our source catalogue has been constructed from all sources which have a peak brightness exceeding our $5 \sigma$ limit of $\sim 50 \mathrm{mJy}$ beam $^{-1}$ at "full" velocity resolution of $26 \mathrm{~km} \mathrm{~s}^{-1}$ and correspondingly fainter brightnesses after velocity smoothing to 52 and $104 \mathrm{~km} \mathrm{~s}^{-1}$. Simulations involving the injection of artificial sources into similar total power H I data-cubes by Rosenberg \& Schneider (2002) have shown that an asymptotic completeness of about $90 \%$ is reached at a signal-to-noise ratio of 8 , while the completeness at a signal-to-noise ratio of 5 is only likely to be about $30 \%$.

We have used the Duchamp (Whiting 2008) source finding tool to find candidate sources in the data cubes at the different velocity resolutions. The source finder was found to be robust down to this $5 \sigma$ limit on peak brightness. All candidate sources were subsequently inspected visually. Artefacts from solar interference or due to bright continuum sources could easily be rejected. A lower threshold for the source finder resulted in a much larger proportion of candidates that were deemed unreliable after visual inspection. Moment maps of all candidate detections have been further inspected and analysed interactively resulting in some additional detections of interesting features. As these features were sought preferentially in the direct vicinity of other galaxies, they have not been cataloged to the same level of completeness throughout the data volume. Although many of these features appear highly significant, their derived properties are often quite sensitive to the form of the spectral baseline that is subtracted.

We have found two classes of objects. The first ones are relatively narrow lines, with a total line width of $\sim 100 \mathrm{~km} \mathrm{~s}^{-1}$ or a $F W H M$ of $\sim 50 \mathrm{~km} \mathrm{~s}^{-1}$ and a peak brightness per $26 \mathrm{~km} \mathrm{~s}^{-1}$ velocity channel that only exceeds the local noise by a factor three or four. The second class of objects has very broad line widths of up to $\sim 500 \mathrm{~km} \mathrm{~s}^{-1}$, however the measured brightness per line channel only exceeds the local noise by a factor two, or not at all. Because of the broad line widths, the integrated detections have a high significance, but such features are particularly difficult to detect with an automatic source finder. Although the detections seem significant, they need further confirmation as the nature and broad line-width of these objects is unexpected. Nevertheless it seems unlikely that these detections are an artefact of the reduction pipeline or the method of bandpass estimation. Only a second order polynomial has been fit to the bandpass 

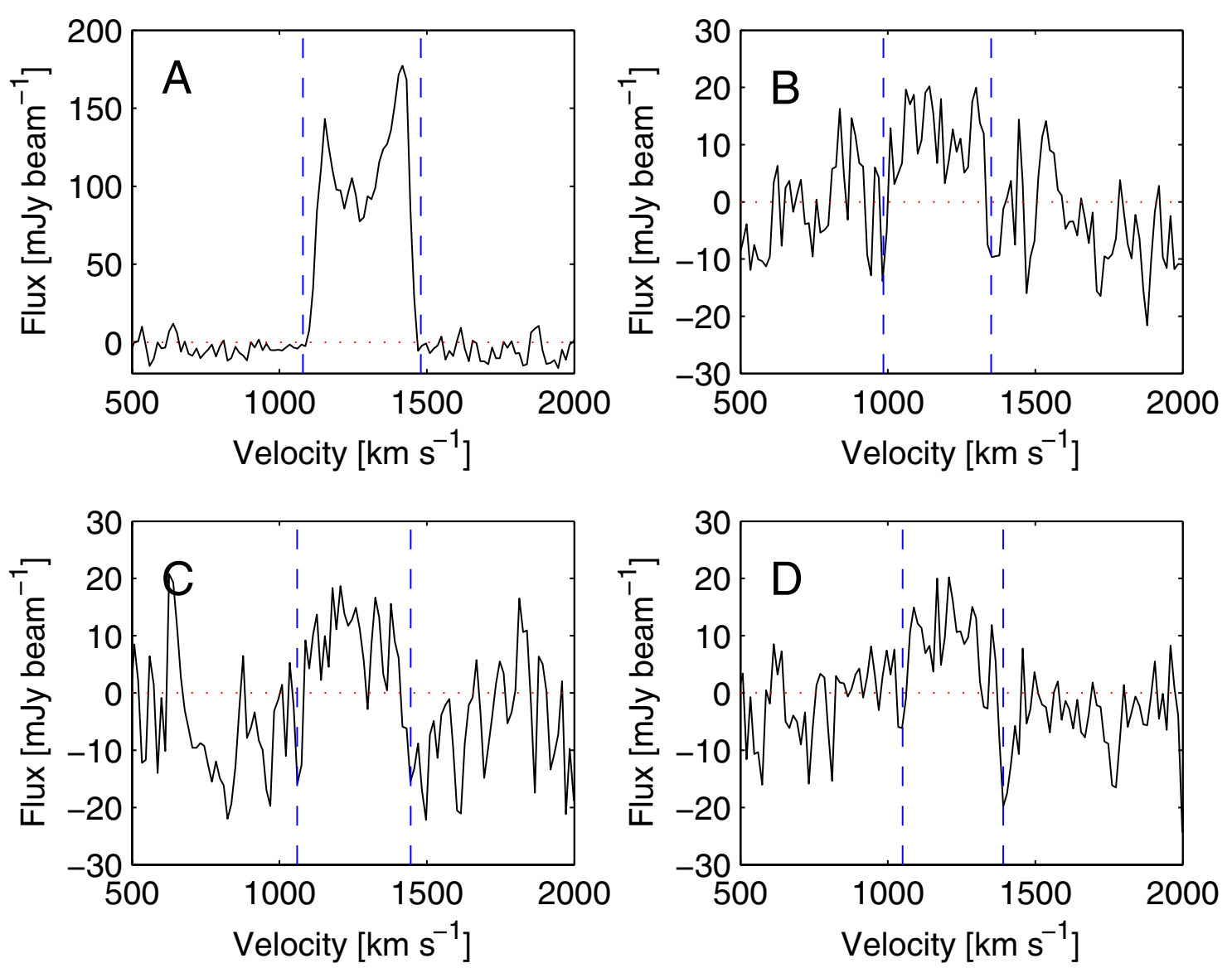

Fig. 7. Line profiles of NGC 3044 (A) and three faint detection (B-D) in the extended environment of the galaxy.

in each spectrum. For most of the broad lines, a range of channels is systematically elevated above the rest of the spectrum and the transition is quite sharp. Higher order polynomials would be needed to artificially create such features.

\section{Discussion}

In this paper we describe how a significant part of the raw HIPASS observations have been reprocessed. The reprocessed region covers the right ascension range from 8 to $17 \mathrm{~h}$ and Declinations from -1 to 10 degrees. A source catalogue and $\mathrm{H}_{\text {I }}$ features without optical counterparts have been presented. Although the original HIPASS product is an excellent one in its own right, by improving the reduction and processing pipeline the quality and number of detections can be significantly improved. The main purpose of reprocessing this particular region is because of the overlap with the WVFS survey as described in Popping \& Braun (2011a) and Popping \& Braun (2011b) and the HIPASS data complements the two data products of the WVFS survey. The first WVFS product has a worse flux sensitivity than the HIPASS data, but a better column density sensitivity due to the very low resolution of this dataset. On the opposite the second WVFS product has a better flux sensitivity than the HIPASS data, but a worse column density sensitivity as the resolution of this data is higher than the resolution of the Parkes telescope.

We will leave detailed analysis and discussion to a later paper, when the three independent datasets will be compared. A few comments will be made that are relevant to this dataset and these detections.

\subsection{Red-shifted $\mathrm{OH}$}

There are several detections, both in the source list, as well as identified as possible filaments, that do not have an optical counterpart. Because there is no optical counterpart, the origin of these features is not straightforward. All detections were considered to be $\mathrm{H}$ I detections that could reside in the vicinity of other objects as tidal remnants or Cosmic Web features. Another scenario that has not been explored is red-shifted $\mathrm{OH}$ emission from sources at a redshift of $z \sim 0.15$. The $1665.401 / 1667.358 \mathrm{MHz}$ doublet of an $\mathrm{OH}$ megamaser emitted at this redshift would have an observed frequency of $\sim 1415 \mathrm{MHz}$. Although confirmed detections of these red-shifted $\mathrm{OH}$ megamasers have not yet been reported, they are predicted to be found in blind Hi surveys. ALFALFA (Giovanelli et al. 2005) expects to find several dozen OHMs in the redshift interval 0.16-0.25. Although the area covered by ALFALFA is significantly larger, based on these numbers we can expect to detect a few OHMs in our survey volume. To detect the $\mathrm{OH}$ doublet, two similar peaks should be identified with a separation of $\sim 350 \mathrm{~km} \mathrm{~s}^{-1}$. When looking at the profiles of known OHMs in e.g. Darling \& Giovanelli (2002), the doublet is not always clearly apparent and so this requirement might be somewhat relaxed. All the documented OHMs do have a broad line-width, typically larger than $300 \mathrm{~km} \mathrm{~s}^{-1}$. This consideration rules out most of our detections without optical counterparts as candidate OHMs, as the line-widths are much narrower. There are however a few cases, where this may be a possible scenario, namely: HRF 0948+0043, HRF 1224+0846, HRF 1225+0826 and HRF $1323+0206$. The other prediction of this scenario is that a suitable ULIRG at $z \sim 0.15$ should be coincident with the $\sim 1415 \mathrm{MHz}$ line detection. We have sought for objects at 
the appropriate redshift that coincide with the spatial positions of these detections, but did not find any sources that could cause redshifted $\mathrm{OH}$ emission.

\subsection{Gas accretion modes}

An interesting question regarding structure formation is how the intergalactic medium fuels the galaxies; i.e. how gas is accreted. The two most discussed scenarios are hot mode and cold mode accretion (Kereš et al. 2005). The line-width of a detection can be used to estimate the upper limit of the kinetic temperature of the gas and is given by:

$$
T_{\text {kin }} \leq \frac{m_{\mathrm{H}} \Delta V^{2}}{8 k \ln 2}
$$

where $m_{\mathrm{H}}$ is the mass of an hydrogen atom, $k$ is the Boltzmann constant and $\Delta V$ is the $\mathrm{H}_{\mathrm{I}}$ line-width at $F W H M$. This equation gives an upper limit to kinetic temperature, as internal turbulence or rotation can also increase the line-width of an object.

In the case of cold mode accretion with temperatures of the order of $T<10^{5} \mathrm{~K}$, the line-widths of the gas are relatively narrow, up to $\sim 100 \mathrm{~km} \mathrm{~s}^{-1}$. The conditions to observe such gas are relatively easily satisfied, the neutral fraction in cold gas is still significant so that the $\mathrm{H}$ i column density is still high. Although it is difficult to distinguish tidal remnants from pristine gas that is fuelling the galaxies, gas accretion is a very plausible scenario. The details of our detections will be discussed later when other data products are included. There are however a handful of detections in the direct vicinity of other galaxies that are not bright, but have line-widths up to $>500 \mathrm{~km} \mathrm{~s}^{-1}$. One possible scenario is that this is red-shifted $\mathrm{OH}$ emission as is discussed in the previous subsection, but this is very unlikely. These line-widths are however also the line-widths that are expected in the case of hotmode-accretion. This is gas that is gradually shock heated during structure formation to virial temperatures and than rapidly cools down to accrete onto the galaxies, for a more extended explanation see Kereš et al. (2005). Because this gas is highly ionised, the neutral fraction is very low, so the $\mathrm{H}$ i component of such gas is extremely small and very unlikely.

\section{Conclusion}

Original data of the H I Parkes All Sky Survey has been reprocessed, that overlaps in sky coverage with the Westerbork Virgo Filament Survey. This region was selected to complement the WVFS and use HIPASS to confirm candidate detections. Furthermore, HIPASS is an excellent dataset, to search for diffuse $\mathrm{H}_{\mathrm{I}}$ features that can be related to the neutral component of the Cosmic Web.

By using an improved reduction strategy, we achieved a reduced rms value and lower artefact level, compared to the original HIPASS product. In the reprocessed data, we achieve a noise value of $\sim 10 \mathrm{mJy}^{-1}$ beam ${ }^{-1}$ over $26 \mathrm{~km} \mathrm{~s}^{-1}$, which is a $\sim 20 \%$ improvement over the original HIPASS product. The data has a $1 \sigma$ brightness sensitivity of $\sim 3.5 \times 10^{17} \mathrm{~cm}^{-2}$, which allows direct detection in $\mathrm{H}_{\mathrm{I}}$ emission of some of the higher column density Lyman Limit Systems seen in QSO absorption line studies.
The major difference with respect to the original HIPASS cubes is that negative artefacts in the bandpass in the vicinity of bright sources are almost completely eliminated. This allows us to search for diffuse and extended $\mathrm{H}_{\mathrm{I}}$ emission, which has not been possible before.

In total we have detected 203 objects in the reprocessed region, of which 29 had not been catalogued in the original HIPASS source catalogue. Fourteen of these detections are completely new H I detections, of which many do not have an optical counterpart. Although these detections are briefly mentioned, detailed discussion and possible confirmation from other data sets will be presented in a subsequent paper in this series.

In this work only a relatively small part of the HIPASS data has been reprocessed, as that survey covers the complete Southern sky and the Northern sky up to +24 degrees in Declination. Apart from this work, steps have been undertaken to further improve the reduction pipeline and reprocess the complete survey area. Improved data cubes, together with improved object-searching algorithms will permit detection of significantly more sources. This will provide improved statistics on the distribution of $\mathrm{H}_{\mathrm{I}}$ in the local universe. The reprocessed cubes will also permit an unbiased search for diffuse neutral hydrogen and Cosmic Web filaments, although currently there is no other all-sky $\mathrm{H}_{\text {I }}$ survey with an appropriate brightness sensitivity and distinct angular resolution to complement this data.

Acknowledgements. We would like to thank several members of the HIPASS team and especially Mark Calabretta for help and useful discussions, that helped in improving the quality of the reduced HIPASS data. The Parkes telescope is part of the Australia Telescope which is funded by the Commonwealth of Australia for operation as a National Facility managed by CSIRO.

\section{References}

Barnes, D. G., Staveley-Smith, L., de Blok, W. J. G., et al. 2001, MNRAS, 322, 486 Braun, R., \& Thilker, D. A. 2004, A\&A, 417, 421

Cen, R., \& Ostriker, J. P. 1999, ApJ, 514, 1

Darling, J., \& Giovanelli, R. 2002, AJ, 124, 100

Davé, R., Hernquist, L., Katz, N., \& Weinberg, D. H. 1999, ApJ, 511, 521

Davé, R., Cen, R., Ostriker, J. P., et al. 2001, ApJ, 552, 473

Giovanelli, R., Haynes, M. P., Kent, B. R., et al. 2005, AJ, 130, 2598

Gooch, R. 1996, in Astronomical Data Analysis Software and Systems V, ed. G. H. Jacoby, \& J. Barnes, ASP Conf. Ser., 101, 80

Kent, B. R., Giovanelli, R., Haynes, M. P., et al. 2008, AJ, 136, 713

Kereš, D., Katz, N., Weinberg, D. H., \& Davé, R. 2005, MNRAS, 363, 2

Lee, S.-W., \& Irwin, J. A. 1997, ApJ, 490, 247

Meyer, M. J., Zwaan, M. A., Webster, R. L., et al. 2004, MNRAS, 350, 1195

Popping, A. 2010, Ph.D. Thesis, University of Groningen

Popping, A., \& Braun, R. 2011a, A\&A, 527, A90

Popping, A., \& Braun, R. 2011b, A\&A, 528, A28

Putman, M. E., Staveley-Smith, L., Freeman, K. C., Gibson, B. K., \& Barnes, D. G. 2003, ApJ, 586, 170

Rosenberg, J. L., \& Schneider, S. E. 2002, ApJ, 567, 247

Sand, D. J., Treu, T., Ellis, R. S., \& Smith, G. P. 2005, ApJ, 627, 32

Spergel, D. N., Bean, R., Doré, O., et al. 2007, ApJS, 170, 377

Whiting, M. T. 2008, Astronomers! Do You Know Where Your Galaxies are?, ed. H. Jerjen, \& B. S. Koribalski, 343

Wong, O. I., Ryan-Weber, E. V., Garcia-Appadoo, D. A., et al. 2006, MNRAS, 371,1855 
A\&A 533, A122 (2011)

\section{Appendix A: Spectra HI detections in the reprocessed HI Parkes All Sky Survey data}
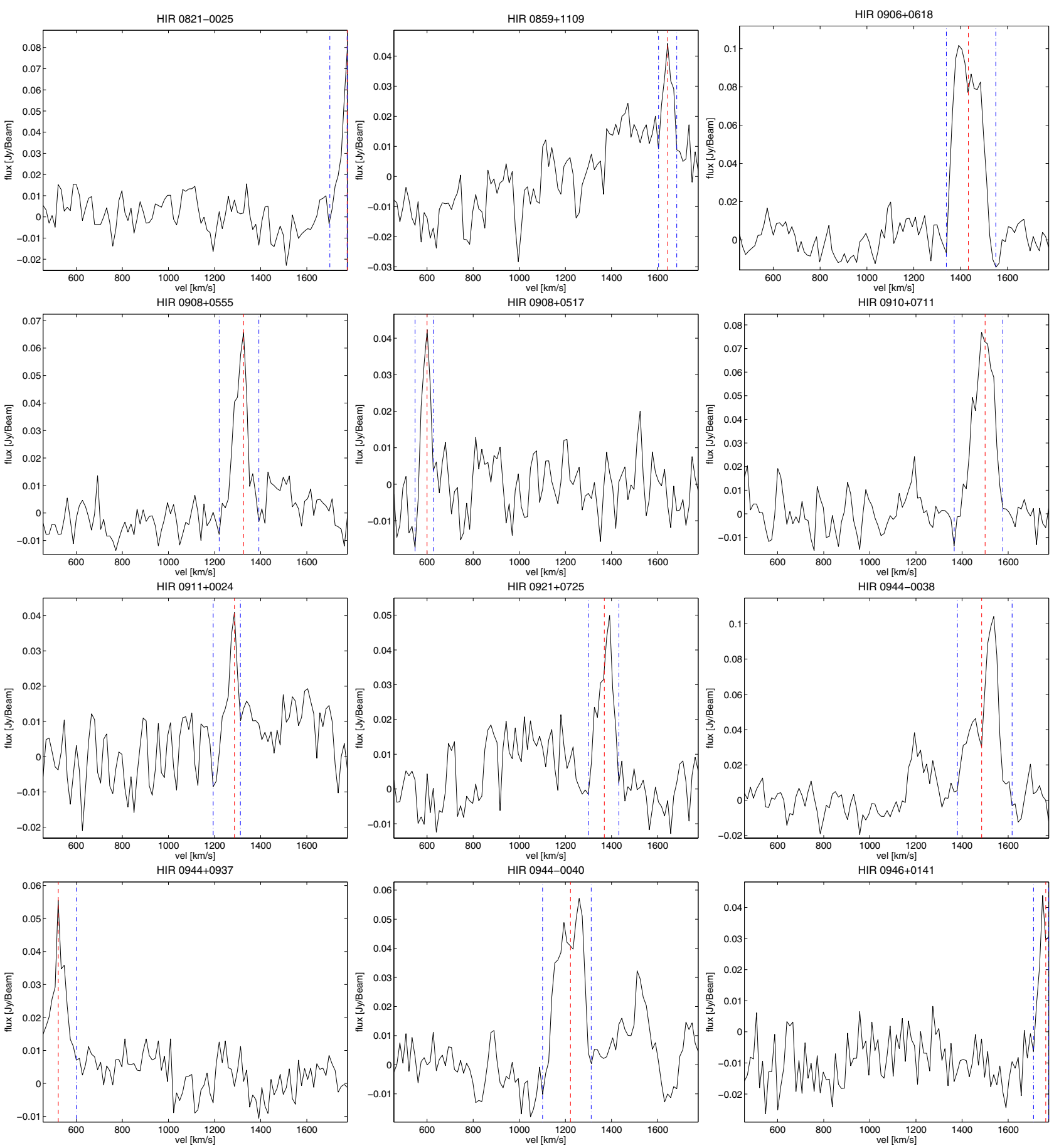

Fig. A.1. Spectra of all detections of neutral hydrogen in the reprocessed region of the H I Parkes All Sky Survey. The velocity width of each object is indicated by the two blue dash-dotted lines, while the central velocity is shown by the red dashed line. 
A. Popping and R. Braun: Diffuse neutral hydrogen in the H I Parkes All Sky Survey
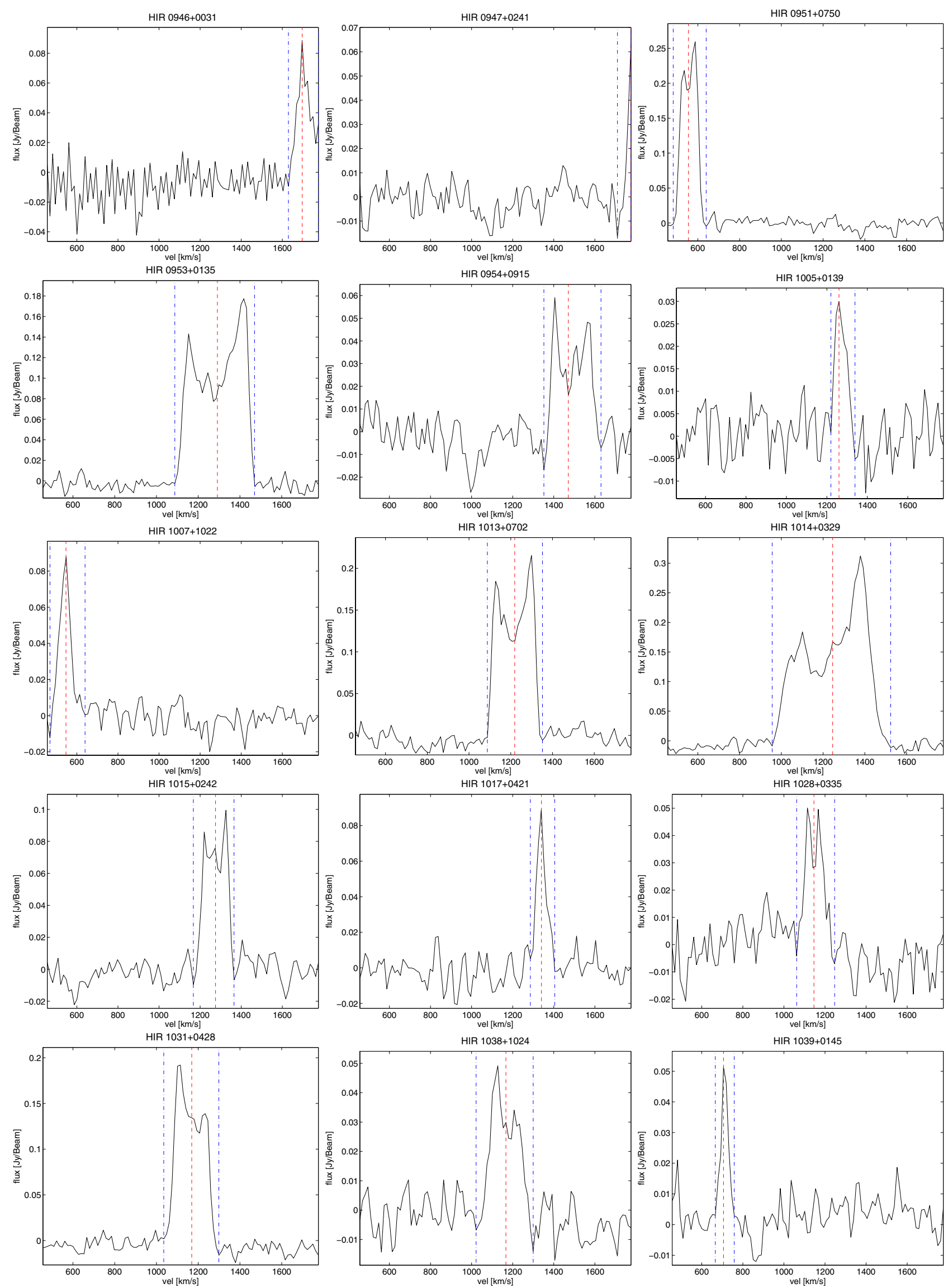

Fig. A.1. continued. 
A\&A 533, A122 (2011)
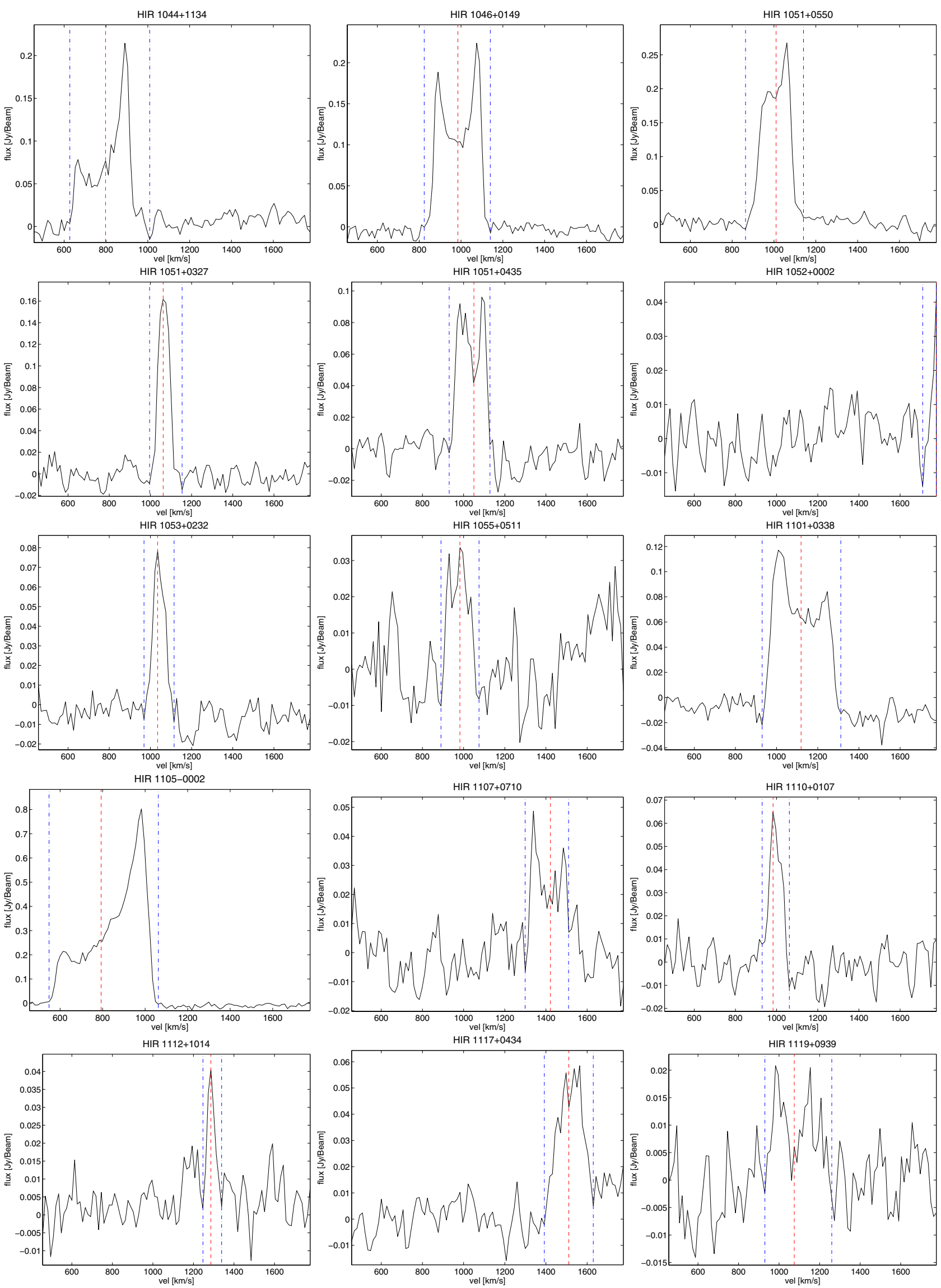

Fig. A.1. continued. 
A. Popping and R. Braun: Diffuse neutral hydrogen in the H I Parkes All Sky Survey
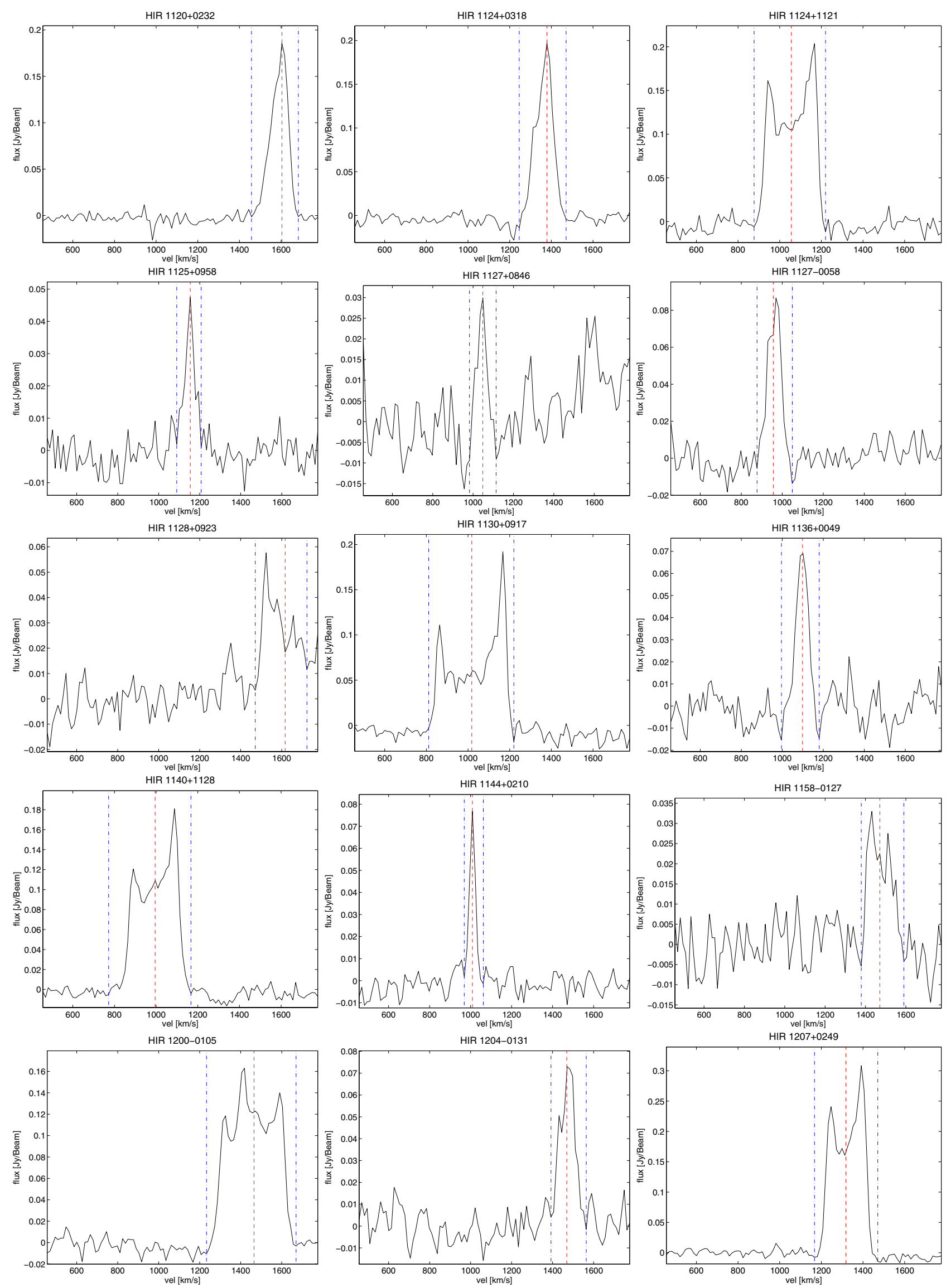

Fig. A.1. continued. 
A\&A 533, A122 (2011)
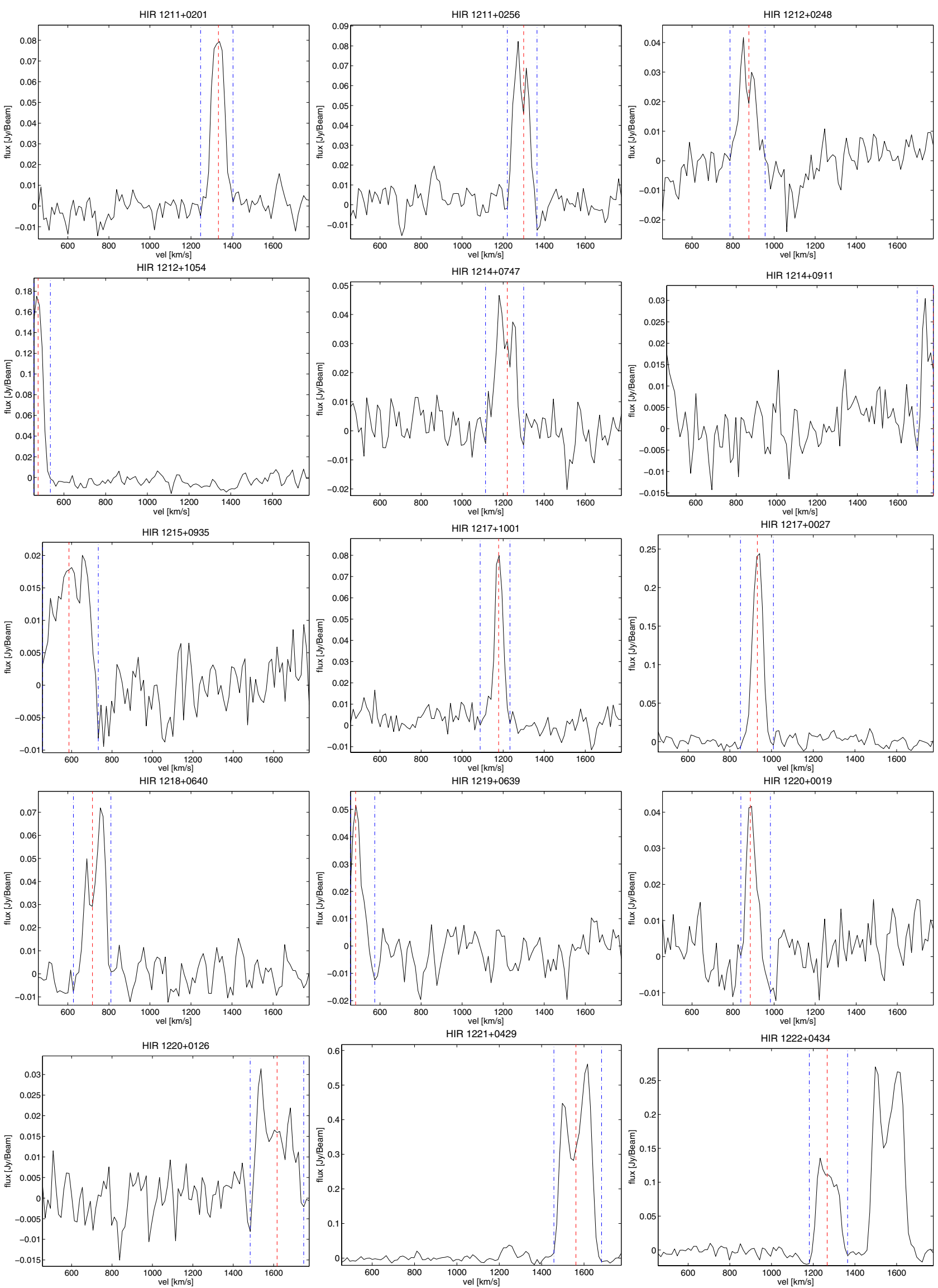

Fig. A.1. continued. 
A. Popping and R. Braun: Diffuse neutral hydrogen in the H I Parkes All Sky Survey
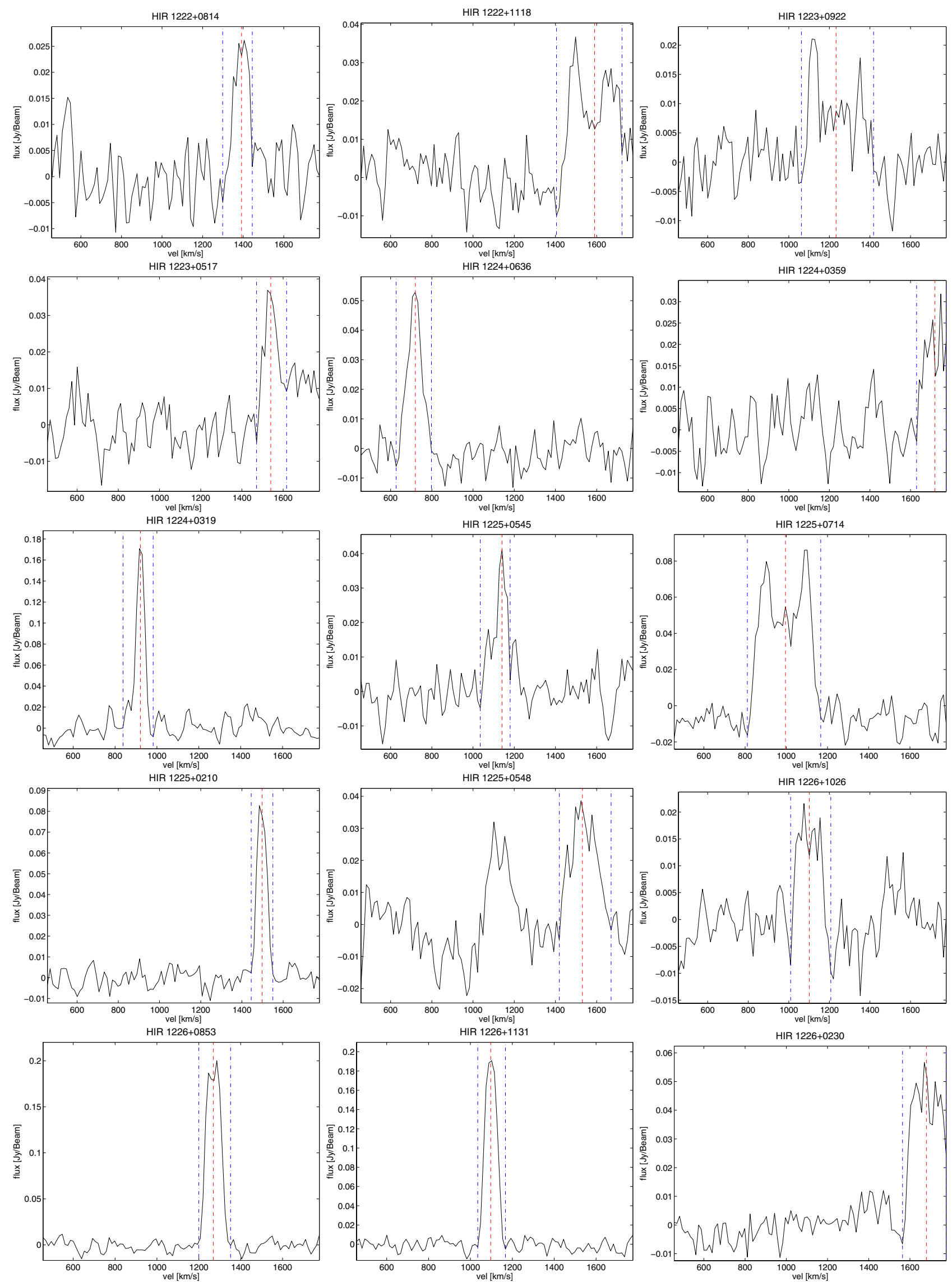

Fig. A.1. continued. 
A\&A 533, A122 (2011)
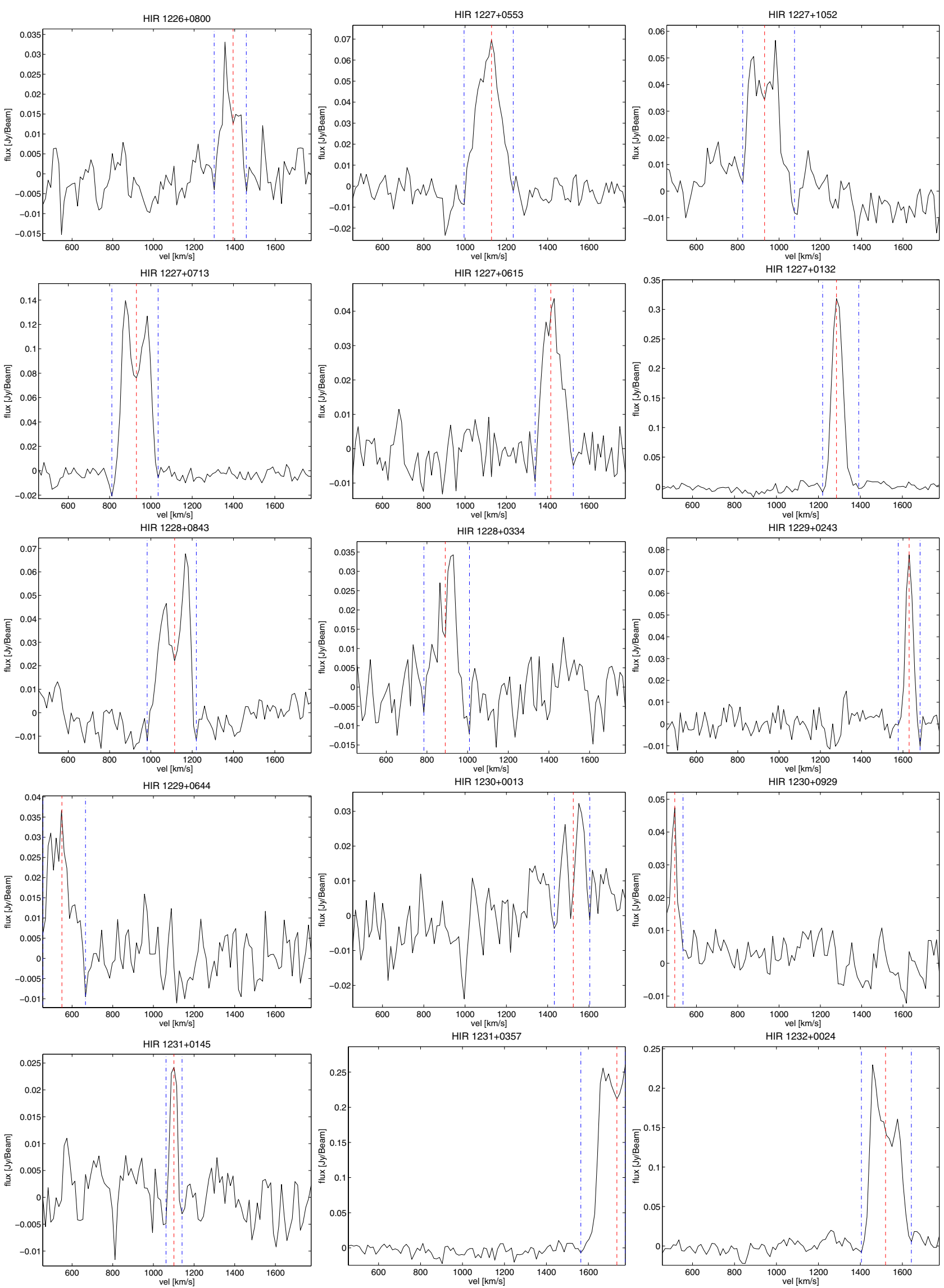

Fig. A.1. continued. 
A. Popping and R. Braun: Diffuse neutral hydrogen in the H I Parkes All Sky Survey
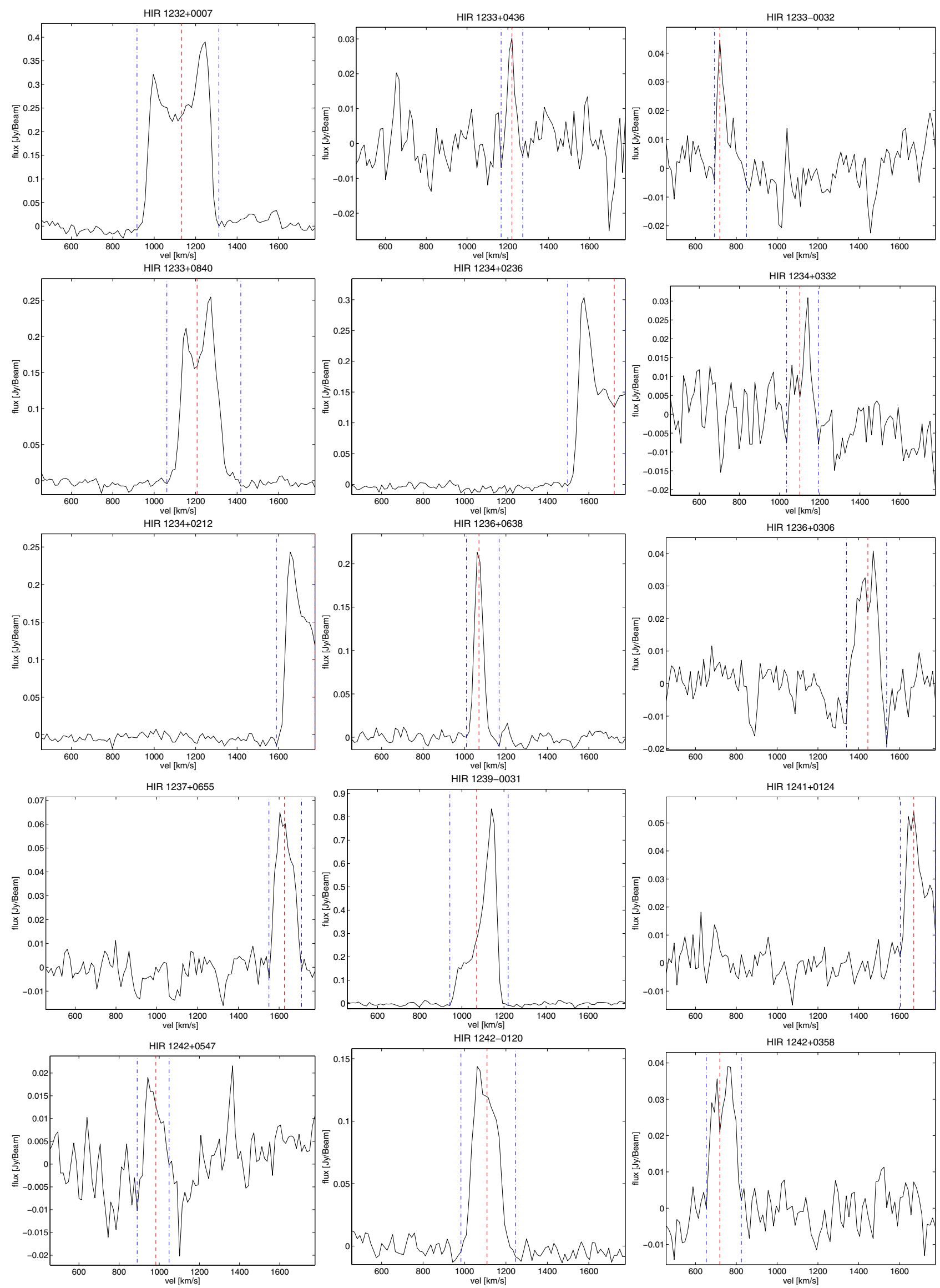

Fig. A.1. continued. 
A\&A 533, A122 (2011)
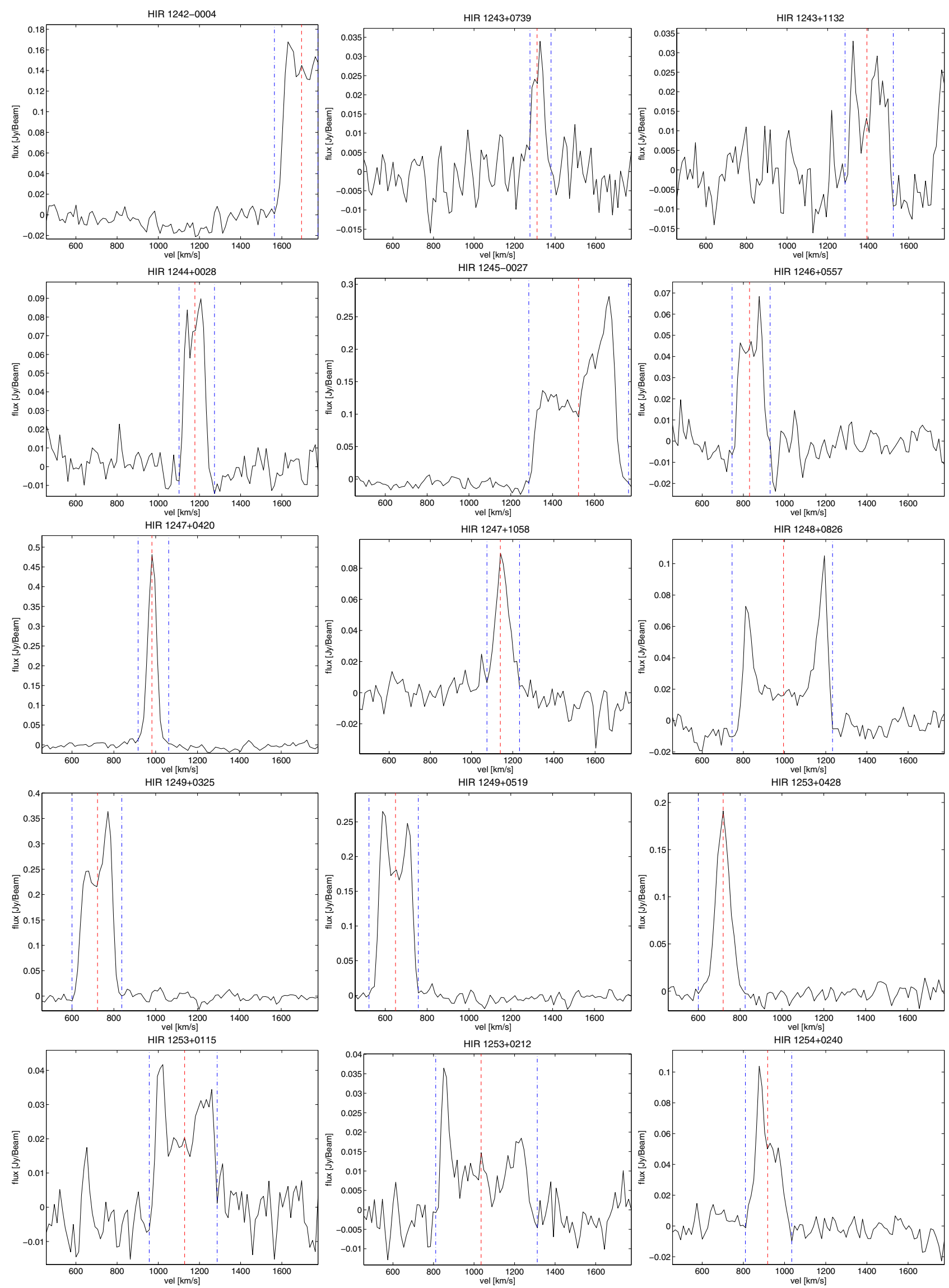

Fig. A.1. continued. 
A. Popping and R. Braun: Diffuse neutral hydrogen in the H I Parkes All Sky Survey
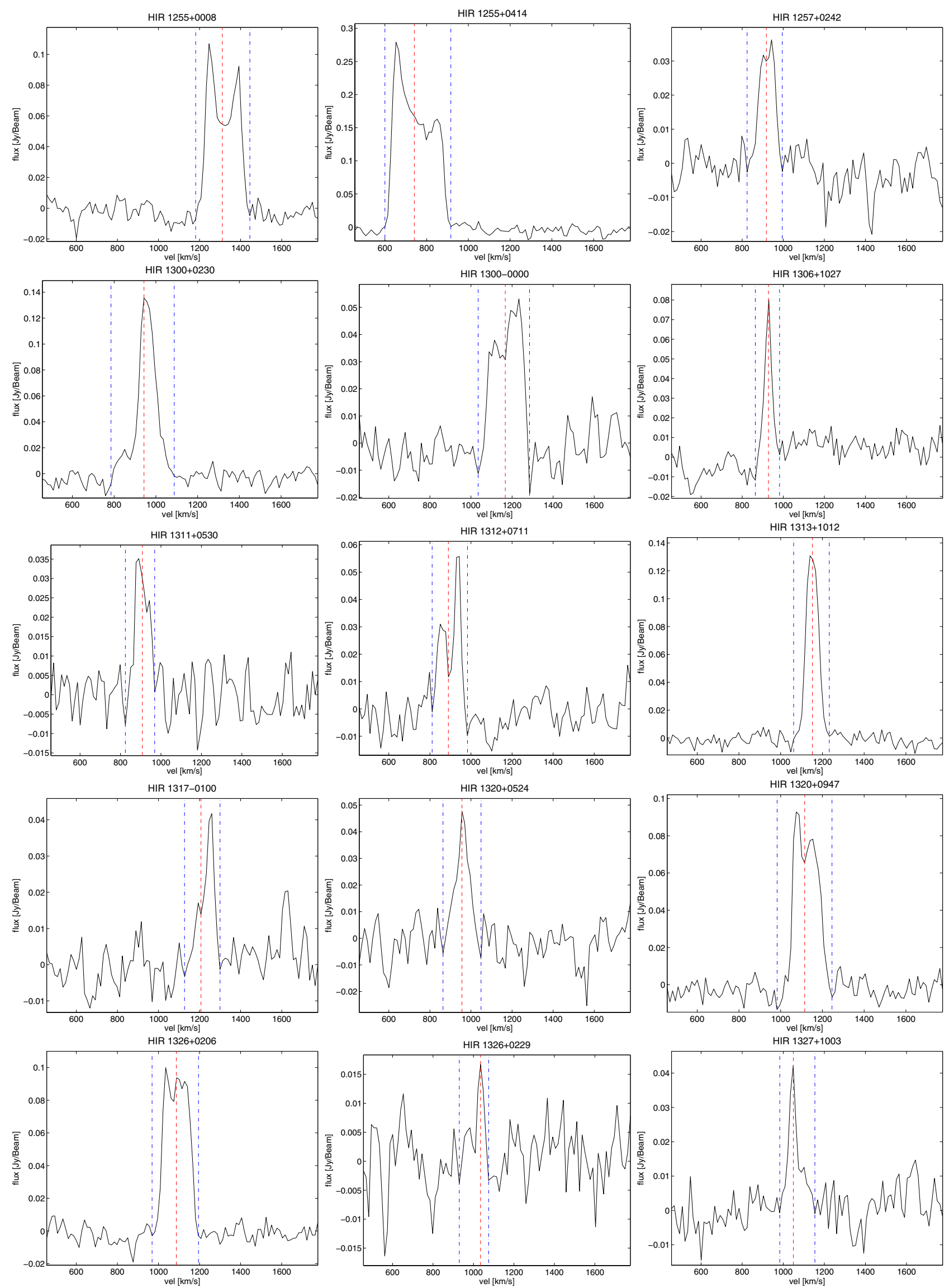

Fig. A.1. continued. 
A\&A 533, A122 (2011)
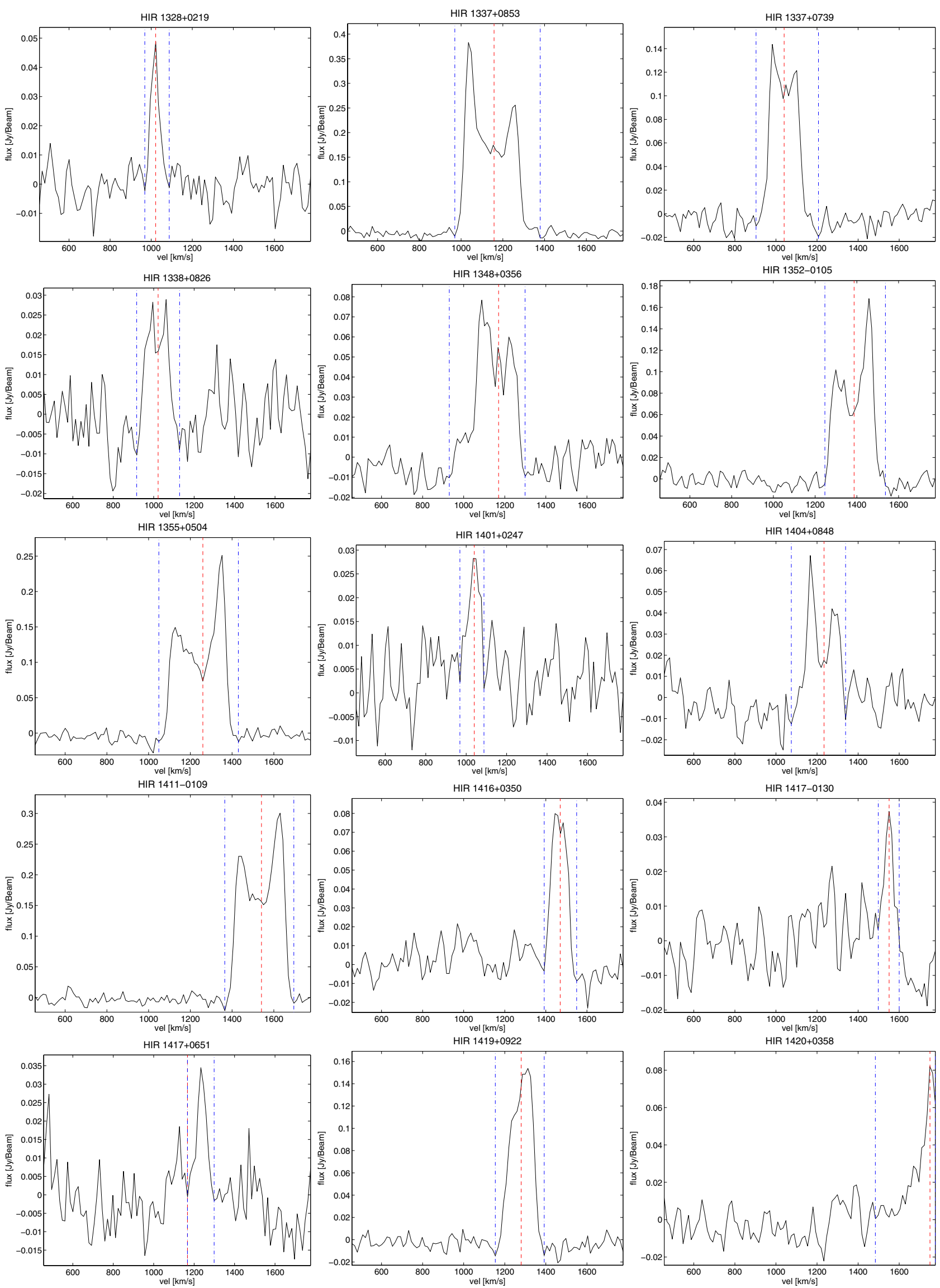

Fig. A.1. continued. 
A. Popping and R. Braun: Diffuse neutral hydrogen in the H I Parkes All Sky Survey
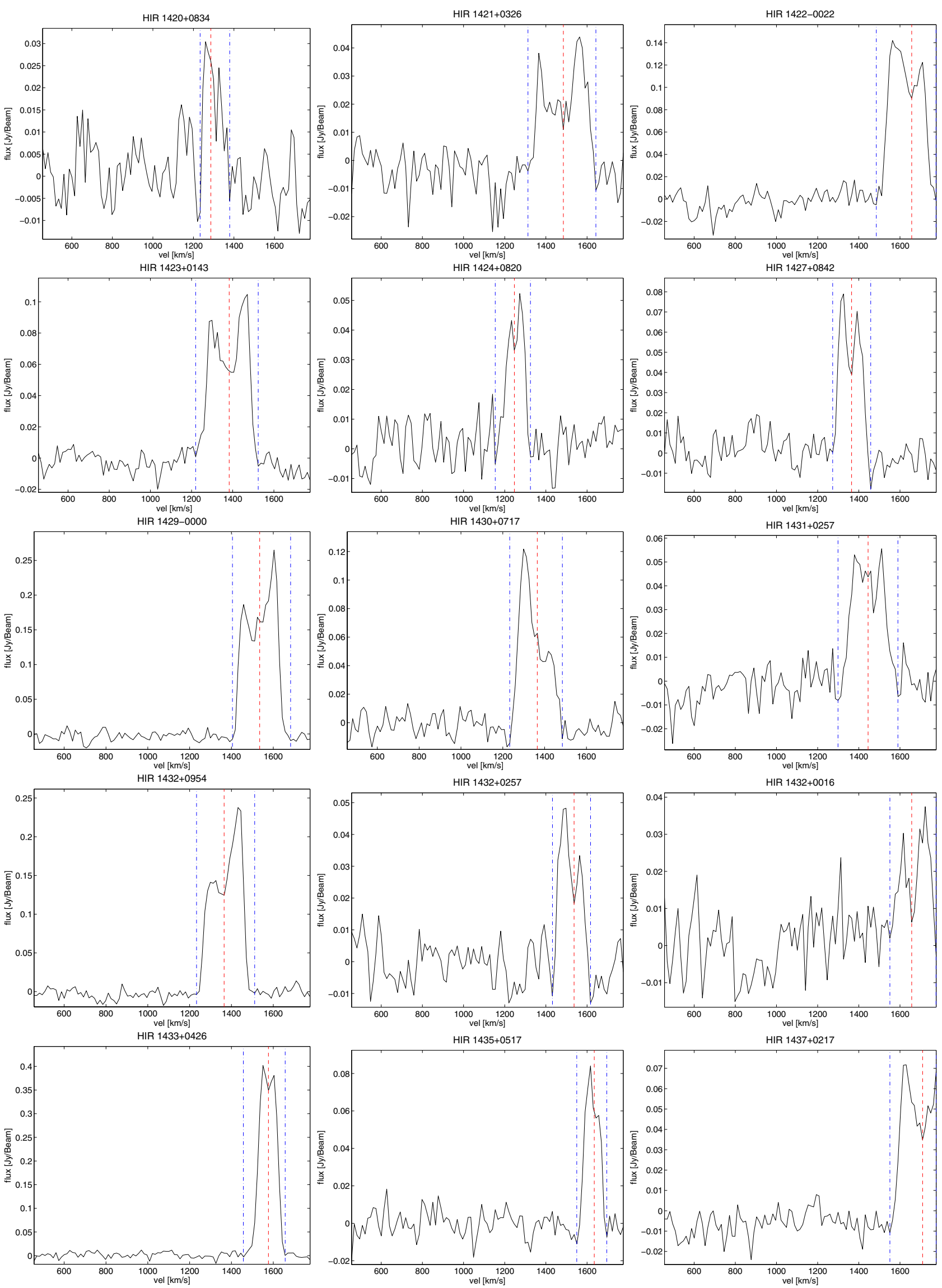

Fig. A.1. continued. 
A\&A 533, A122 (2011)
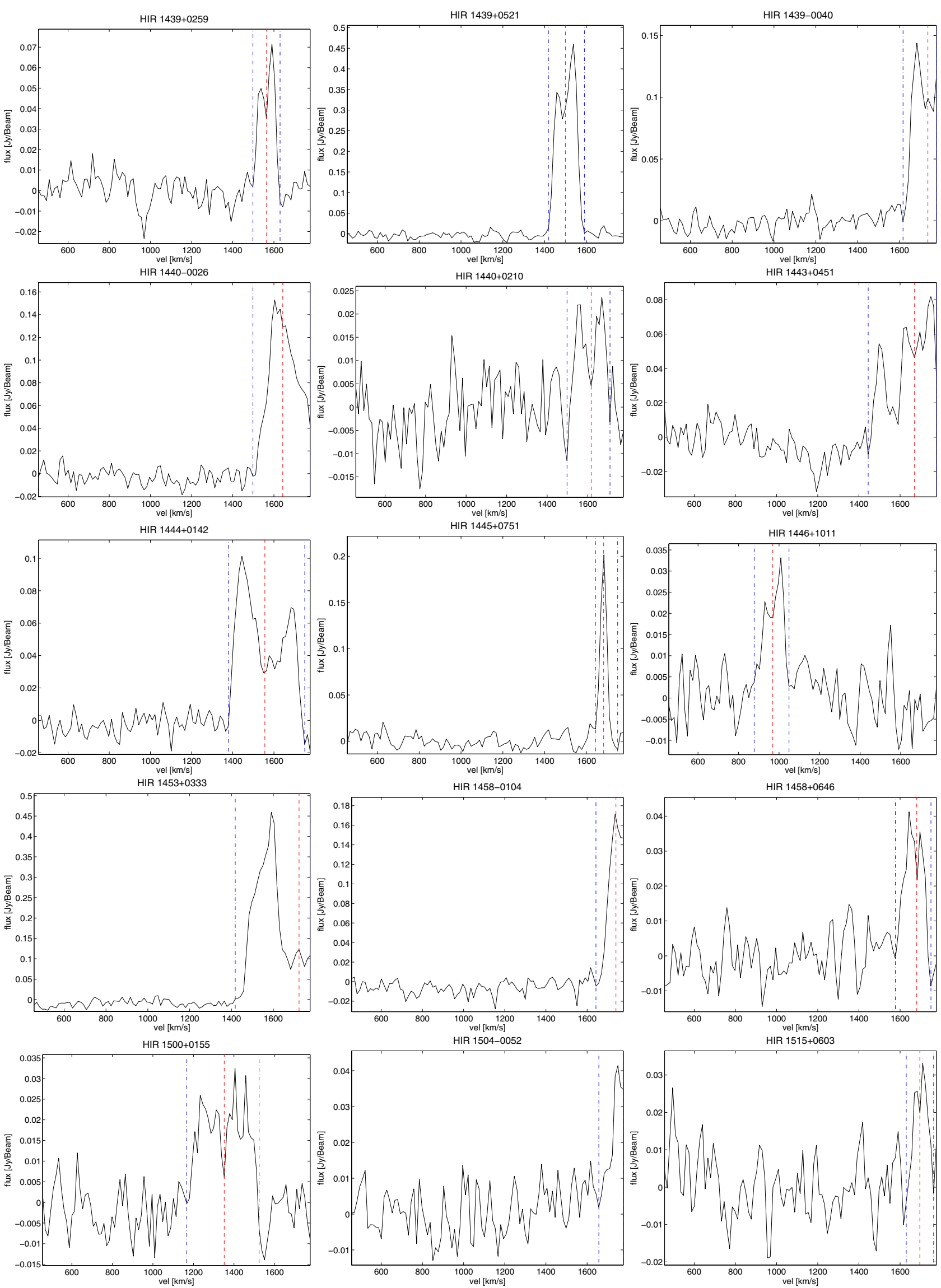

Fig. A.1. continued. 
A. Popping and R. Braun: Diffuse neutral hydrogen in the H I Parkes All Sky Survey
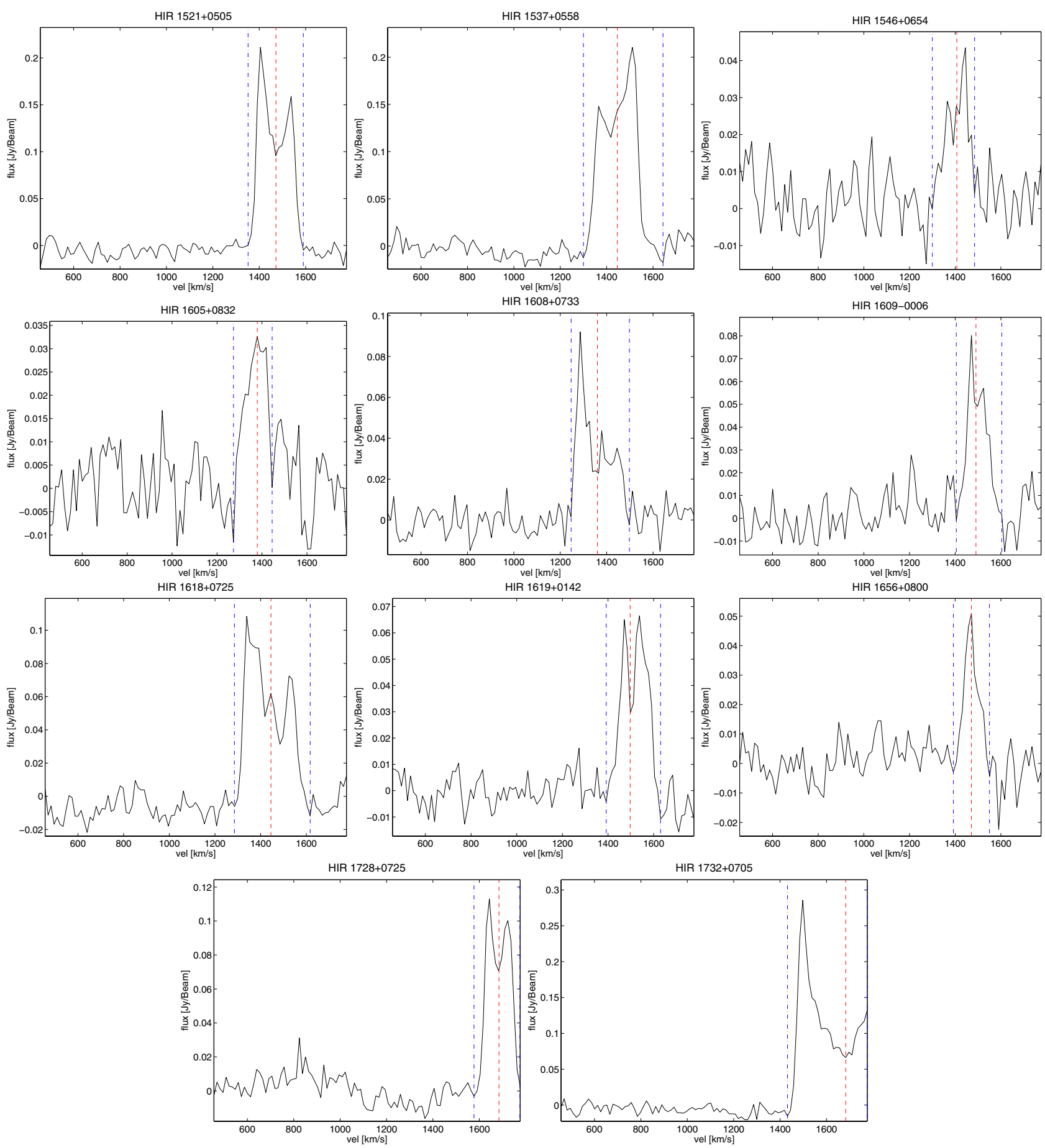

Fig. A.1. continued. 